\title{
Global bifurcations in the two-sphere: a new perspective
}

\author{
Yu. Ilyashenko*ł\$ \\ Yu. Kudryashov* *ił \\ I. Schurov*¥ף \\ August 18, 2018
}

\begin{abstract}
We construct an open set of structurally unstable three parameter families whose weak and so called moderate topological classification defined below has a numerical invariant that may take an arbitrary positive value. Here and below "families" are "families of vector fields in the two-sphere". This result disproves an Arnold's conjecture of 1985. Then we construct an open set of six parameter families whose moderate topological classification has a functional invariant. This invariant is an arbitrary germ of a smooth map $\left(\mathbb{R}_{+}, a\right) \rightarrow\left(\mathbb{R}_{+}, b\right)$. More generally, for any positive integers $d$ and $d^{\prime}$, we construct an open set of families whose topological classification has a germ of a smooth map $\left(\mathbb{R}_{+}^{d}, a\right) \rightarrow\left(\mathbb{R}_{+}^{d^{\prime}}, b\right)$ as an invariant. Any smooth germ of this kind may be realized as such an invariant. These results open a new perspective of the global bifurcation theory in the two sphere. This perspective is discussed at the end of the paper.
\end{abstract}

\section{Introduction: structurally unstable families}

There are many families of planar vector fields whose bifurcations are investigated up to now. All of them are weakly structurally stable in their domains (in a neighborhood of a singular point or a polycycle), in a sense explained below. In 1985 Arnold suggested a perspective of the development of the global bifurcation theory in the two sphere [1]. In particular, he conjectured that generic families of vector fields considered on the whole sphere are structurally stable. The first result of this paper disproves this conjecture.

Arnold includes the conjecture mentioned above in the list of six. Right after the statement of these conjectures Arnold writes:

\footnotetext{
*National Research University Higher School of Economics, Russia

†Cornell University, US

${ }^{\ddagger}$ Independent University of Moscow

$\S$ The authors were supported in part by the grant RFBR 16-01-00748

『Research of I.S. was supported in part by Dynasty Foundation
} 
"Certainly proofs or counterexamples to the above conjectures are necessary for investigating nonlocal bifurcations in generic l-parameter families."

The current paper is motivated by the Arnold's conjectures. As we show, the most nontrivial of them appeared to be wrong. This opens a new perspective of the development of the global bifurcation theory in the two sphere. It is discussed in Section 5.

\subsection{Basic definitions and notation}

Recall the necessary definitions. We give them in the general setting, though for our needs everywhere below we may take $M=S^{2}$. Here and below $B \subset \mathbb{R}^{k}$ (base of a family) is a topological open ball.

Denote by $\operatorname{Vect}(M)$ the set of $C^{3}$-smooth vector fields on $M$.

Definition 1. A family of vector fields on a manifold $M$ with the base $B$ is a vector field $V$ on $B \times M$ tangent to the fibers $\{\alpha\} \times M, \alpha \in B$. The dimension of a family is the dimension of its base.

An equivalent definition.

Definition 2. A family of vector fields on $M$ with the base $B$ is a smooth map $V$ : $B \rightarrow \operatorname{Vect}(M)$.

The equivalence is obvious. Denote by $\mathcal{V}_{k}(M)$ the space of $k$-parameter families of vector fields on $M$ which are $C^{3}$ smooth as vector fields on $B \times M$.

Definition 3. A family of vector fields is transversal to a Banach submanifold $\mathbf{T}$ of $\operatorname{Vect}(M)$ provided that the corresponding map $V: B \rightarrow \operatorname{Vect}(M)$ is transversal to $\mathbf{T}$.

Definition 4. Two vector fields $v$ and $\tilde{v}$ on a manifold $M$ are called orbitally topologically equivalent, if there exists a homeomorphism $M \rightarrow M$ that links the phase portraits of $v$ and $\tilde{v}$, that is, sends orbits of $v$ to orbits of $\tilde{v}$ and preserves their time orientation.

Definition 5. Two families of vector fields $\left\{v_{\alpha} \mid \alpha \in B\right\},\left\{\tilde{v}_{\tilde{\alpha}} \mid \tilde{\alpha} \in \tilde{B}\right\}$ on $M$ are called weakly topologically equivalent if there exists a map

$$
H: B \times M \rightarrow \tilde{B} \times M, \quad H(\alpha, x)=\left(h(\alpha), H_{\alpha}(x)\right)
$$

such that $h: B \rightarrow \tilde{B}$ is a homeomorphism, and for each $\alpha \in B$ the map $H_{\alpha}: M \rightarrow M$ is a homeomorphism that links the phase portraits of $v_{\alpha}$ and $\tilde{v}_{h(\alpha)}$.

Two families are topologically equivalent if there exists a homeomorphism $H$ with above properties. Topological classification of families with a very simple dynamics may have functional invariants that occur due to the requirement of the continuity of $H_{\alpha}$ in $\alpha[10]$. Thus, the topological equivalence is too rigid. On the other hand, weak topological equivalence introduced in [1] is too lousy: some families with apparently different bifurcations occur to be weakly topologically equivalent. For this reason we introduce here a new equivalence relation of moderate topological equivalence. This 
relation is needed and defined in this paper for vector fields having hyperbolic singular points only.

For a family $V$ of vector fields, denote by $\operatorname{Sing} V$ the set of all the singular points of the family, by Per $V$ the union of all the limit cycles, and by $\operatorname{Sep} V$ the union of all the separatrixes of the vector fields of the family.

Definition 6. We say that $V$ and $\tilde{V}$ are moderately topologically equivalent provided that there exists a linking map $H$, see (1), which is continuous in $(\alpha, x)$ at the set $\overline{\operatorname{Sing} V \cup \operatorname{Per} V \cup \operatorname{Sep} V}$.

Sometimes the parameter value $\alpha=0$ is distinguished in some way. Then we also consider a local version of the equivalence relation above.

Definition 7. Two families $V$ and $\tilde{V}$ are locally moderately topologically equivalent at $\alpha=0$ provided that there exists a linking map $H$, see (1), which is continuous in $(\alpha, x)$ at the set $\overline{\operatorname{Sing} V \cup \operatorname{Per} V \cup \operatorname{Sep} V} \cap\{\alpha=0\}$.

A definition of moderate topological equivalence for general families of vector fields is given in [4], work in progress. It requires more technical details that we skip here.

Definition 8. We say that a family of vector fields is moderately (weakly) structurally stable if it is moderately (weakly) topologically equivalent to its small perturbations.

\subsection{Main results}

Our main results are

Theorem 1. There exists a non-empty open subset of $\mathcal{V}_{3}\left(S^{2}\right)$ such that each family from this set is moderately structurally unstable. Moreover, moderate topological classification of these families has numeric invariant that may take any positive value.

Theorem 2. There exists a non-empty open subset of $\mathcal{V}_{6}\left(S^{2}\right)$ such that the moderate topological classification of families from this set has a functional invariant, namely a germ of a function $f:\left(\mathbb{R}_{+}, a\right) \rightarrow\left(\mathbb{R}_{+}, b\right)$. Moreover, any such germ for any positive $a$, $b$ may be realized as an invariant of this classification.

More generally,

Theorem 3. For any positive integers $d, d^{\prime}$ there exists an open subset of $\mathcal{V}_{k}\left(S^{2}\right), k=$ $3 d+2 d^{\prime}+1$, such that the moderate topological classification of families from this set has a functional invariant, namely a germ of a map $f:\left(\mathbb{R}_{+}^{d}, a\right) \rightarrow\left(\mathbb{R}_{+}^{d^{\prime}}, b\right)$. Moreover, any such germ for any positive vectors $a \in \mathbb{R}_{+}^{d}, b \in \mathbb{R}_{+}^{d^{\prime}}$ may be realized as an invariant of this classification.

Theorem 2 is a corollary of Theorem 3 , but we state and prove it separately because it is simpler and its proof contains all the main ideas needed for the proof of the more general result. These theorems hold true if the moderate equivalence in their statement is replaced by the weak equivalence. The proofs of these modified theorems require more technical details, and we skip them. 


\section{Numerical invariants}

\subsection{A special class of degenerate vector fields of codimension three}

In this section we prove Theorem 1 modulo so called Asymptotic Lemma proved in Section 4. We will first describe the degenerate vector fields, then their unfoldings.

\subsubsection{Polycycles in the sphere}

We do not recall here the detailed definitions of polycycles and their monodromy maps; they may be found in [6]. We only mention that a hyperbolic polycycle is a separatrix polygon whose vertexes are hyperbolic saddles, and edges are saddle connections. The monodromy (or Poincaré map) $\Delta_{\gamma}$ along a polycycle $\gamma$, if exists, is defined like the Poincaré map of a cycle. The only difference is that the map $\Delta_{\gamma}$ is defined on a half interval, called a semi-transversal, with the vertex on $\gamma$, rather than on an interval. If the monodromy map of the polycycle is well defined, then the polycycle is called monodromic; if the corresponding semi-transversal points outside (inside) $\gamma$, then we say that $\gamma$ is monodromic from the exterior (from the interior).

Remark 1. Interior and exterior domains on the sphere are to be specified. We represent the sphere $S^{2}$ as $\mathbb{R}^{2} \cup\{\infty\}$. We consider vector fields that have an attracting fixed point $A$ surrounded by a repelling hyperbolic limit cycle $\gamma_{\infty}$ having no other singular points in the domain bounded by $\gamma_{\infty}$ that contains $A$. We suppose that such a point is unique, and place it to infinity. To distinguish the limit cycle $\gamma_{\infty}$, we will call it limit cycle near infinity. Such a vector field on $S^{2}$ will be called normalized. If two normalized vector fields are orbitally topologically equivalent, then the linking homeomorphism brings $\infty$ to $\infty$. For any Jordan curve on $\mathbb{R}^{2}=S^{2} \backslash\{\infty\}$, the exterior domain is the one that contains $\infty$. For a polycycle in $\mathbb{R}^{2}$ represented as a union of Jordan curves, the interior domain is the union of interior domains of these curves.

In what follows, we consider vector fields close to a fixed normalized vector field. Clearly, such vector field is orbitally topologically (and even smoothly) equivalent to a normalized vector field. Therefore, we can normalize a family so that all vector fields of the normalized family are normalized. We shall always assume that our families are normalized.

\subsubsection{A special polycycle of codimension three}

Consider a (normalized) vector field $v$ which has a polycycle $\gamma$ with two vertexes and three edges, see Figure 1. The vertexes are hyperbolic saddles $L$ and $M$ with the characteristic numbers $\lambda$ and $\mu$. Recall that the characteristic number of a saddle is the modulus of the ratio of its eigenvalues, the negative one in the numerator. Suppose that

$$
\lambda<1, \quad \lambda^{2} \mu>1 .
$$

The edges are: a time oriented separatrix loop $l$ of $L$, and two time oriented saddle connections: $L M$ and $M L$, see Figure 1. These two connections form a polycycle 
"heart": two other separatrixes of $M$ are inside, and those of $L$ are outside this polycyle. The polycycle $\gamma$ is monodromic from the exterior, and the loop $l$ from the interior. The polycycle "heart" is not monodromic at all.

A polycycle $\gamma$ that satisfies these assumptions is called a polycycle of type TH ( $H$ of "heart", and $T$ of "tear" that resembles the separatrix loop $l$ ).

\subsubsection{Vector fields of class $\mathrm{T}$}

Suppose that the vector field $v$ described above has a saddle $E$ outside the polycycle $\gamma$ and a saddle $I$ inside the separatrix loop $l$ of $L$. The letters $E$ and $I$ come from the words "exterior" and "interior". Suppose that one of the unstable separatrixes of E winds onto $\gamma$, and one of the stable separatrixes of $I$ winds onto $l$ in the negative time. Denote these separatrixes by $W_{E}^{u}$ and $W_{I}^{s}$. In Section 4 we prove that inequalities (2) imply the possibility of this winding, see Remark 12. Polycycles described above may occur in generic three-parameter families. Existence of separatrixes $W_{E}^{u}$ and $W_{I}^{s}$ winding to $\gamma$ and from $l$ does not increase the codimension of the degeneracy.

Suppose that there exists a smooth oriented arc $\Gamma$ that goes from $I$ to $E$, intersects $\gamma$ at a unique point $O$ and is transversal to $v$ strictly between its ends. Denote by $\Gamma^{+}, \Gamma^{-}$half open $\operatorname{arcs}$ of $\Gamma$ between $O$ and $E$ ( $E$ excluded), between $O$ and $I$ ( $I$ excluded) respectively. The germs of the monodromy maps $\Delta_{\gamma}:\left(\Gamma^{+}, O\right) \rightarrow\left(\Gamma^{+}, O\right), \Delta_{l}:\left(\Gamma^{-}, O\right) \rightarrow\left(\Gamma^{-}, O\right)$ along the polycycle $\gamma$ and the loop $l$ are well defined. Suppose that the germs $\Delta_{\gamma}, \Delta_{l}^{-1}$ may be extended to the monodromy maps

$$
\Delta_{\gamma}: \Gamma^{+} \rightarrow \Gamma^{+}, \quad \Delta_{l}^{-1}: \Gamma^{-} \rightarrow \Gamma^{-}
$$

that have no fixed points except for $O$.

Assumption 1. The vector field $v$ has exactly two saddles, namely $E$ and $I$, with the following properties: a separatrix of the first saddle winds towards the polycycle $\gamma$; a separatrix of the second saddle winds towards the loop $l$ in the negative time. That is, no other saddle has one of these properties. Moreover, all the singular points of the vector field $v$ are hyperbolic.

The set of vector fields with these properties is called class $\mathbf{T}$.

Denote by $\mathbf{T}^{\circ}$ the set of vector fields $v \in \mathbf{T}$ that have no saddle connections, except for the edges of the polycycle $\gamma$. In particular, a vector field $v \in \stackrel{\circ}{\mathbf{T}}$ has a unique polycycle homeomorphic to $\gamma$. As we shall show in Section 2.2.3, the set $\mathbf{\mathbf { T }}$ is a Banach submanifold of $\operatorname{Vect}\left(S^{2}\right)$ of codimension 3. Let $\mathbf{C}$ be the class of 3-parameter families $V$ such that

- $V$ has a unique intersection $v$ with $\mathbf{T}$;

- $v \in \stackrel{\circ}{\mathbf{T}}$

- $V$ is transversal to $\stackrel{\circ}{\mathbf{T}}$ at $v$, see Definition 3.

Denote by $\mathcal{H}$ (of hyperbolic) the class of vector fields $v \in \operatorname{Vect}\left(S^{2}\right)$ such that 


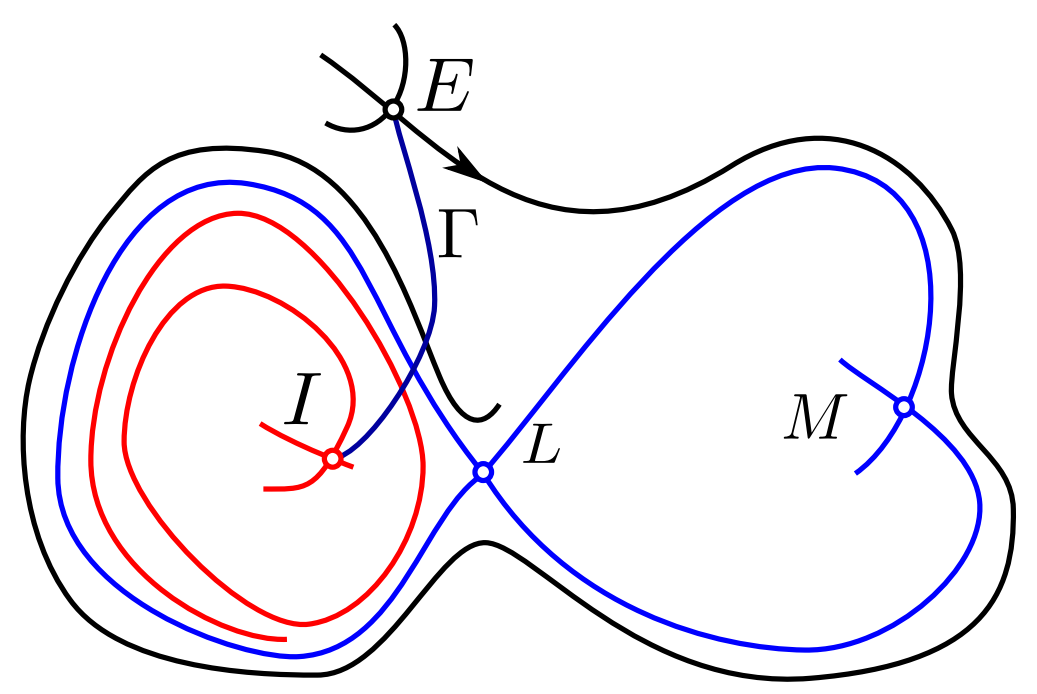

Figure 1: The phase portrait of the degenerate vector field $v$

- all singular points of $v$ are hyperbolic;

- the characteristic number of any saddle of $v$ is different from 1 , and from the inverse square of the characteristic number of any other saddle of $v$.

Obviously, $\mathcal{H}$ is open and dense in $\operatorname{Vect}\left(S^{2}\right)$, and $\mathcal{H} \cap \mathbf{T}$ is dense in $\mathbf{T}$.

Consider a family $V \in \mathbf{C}$ intersecting $\mathbf{T}$ at $v \in \stackrel{\circ}{\mathbf{T}}$. Let $\gamma$ be the polycycle of $v$ of the type $T H$; let $\lambda, \mu$ be the characteristic numbers of the saddles $L, M$, respectively. Let us define

$$
\nu(V)=\frac{-\log \lambda}{\log \lambda \mu^{2}} .
$$

The following theorem is a refinement of Theorem 1 .

Theorem 4. The number $\nu$ given by (4) is a numeric invariant of the moderate classification of families $V \in \mathbf{C}$ such that $V \subset \mathcal{H}$, i.e. for two moderately equivalent families $V, \tilde{V} \in \mathbf{C}$ such that $V, \tilde{V} \subset \mathcal{H}$, we have

$$
\nu(V)=\nu(\tilde{V})
$$

The ratio $\nu$ given by (4) may be expressed through $\frac{\log \lambda}{\log \mu}$. In view of (2), the latter ratio takes the values in $\left(-\frac{1}{2}, 0\right)$. The ratio $\nu$ may take arbitrary positive values.

Theorem 1 follows from Theorem 4, so it is enough to prove the latter theorem.

We introduced the class $\mathcal{H}$ because $\mathbf{T}$ is topologically distinguished in this set.

Proposition 1. Consider two orbitally topologically equivalent vector fields $v, \tilde{v} \in \mathcal{H}$. If $v \in \mathbf{T}$, then $\tilde{v} \in \mathbf{T}$. Moreover, a homeomorphism provided by Definition 4 sends the polycycle $\gamma$, and the saddles $L, M, E, I$ of the vector field $v$ to similar objects for $\tilde{v}$. 
Proof. Let $H_{0}$ be a homeomorphism provided by Definition 4 Let $\tilde{L}, \tilde{M}, \tilde{E}, \tilde{I}, \tilde{l}, \tilde{\gamma}$, $\tilde{W}_{I}^{s}, \tilde{W}_{E}^{u}, \tilde{\Gamma}$ be the images of the corresponding objects for $v$ under $H_{0}$. Since $\tilde{v} \in \mathcal{H}$, the singular points $\tilde{L}, \tilde{M}, \tilde{E}, \tilde{I}$ are hyperbolic saddles for $\tilde{v}$. Next, $\tilde{\gamma}$ is a polycycle homeomorphic to $\gamma$, and $\tilde{W}_{I}^{s}$ (resp., $\tilde{W}_{E}^{u}$ ) is a separatrix of $\tilde{I}$ (resp., $\tilde{E}$ ) winding onto $l$ (resp., $\gamma$ ) in negative (resp., positive) time. Obviously, $\tilde{E}, \tilde{I}$ are unique saddles whose separatrixes wind onto $\gamma, l$. Indeed, if $\tilde{W}_{E}^{\prime u}$ is another separatrix winding onto $\gamma$, then its preimage under $H_{0}$ winds onto $\gamma$, which contradicts Assumption 1 .

Finally, $\tilde{\gamma}$ satisfies all requirements of class $\mathbf{T}$, possibly except for $(2)$. Let $\tilde{\lambda}, \tilde{\mu}$ be the characteristic numbers of $\tilde{L}, \tilde{M}$. Suppose that $\tilde{\lambda} \nless 1$. By definition of $\mathcal{H}$, we have $\tilde{\lambda} \neq 1$, $\tilde{\lambda}^{2} \tilde{\mu} \neq 1$, hence $\tilde{\lambda}>1$. Due to Remark 12 below, this implies that $\tilde{W}_{I}^{s}$ cannot wind onto $\tilde{l}$ in the negative time. Analogously, $\lambda^{2} \tilde{\mu} \ngtr 1$ implies $\tilde{\lambda}^{2} \tilde{\mu}<1$, hence $\tilde{W}_{E}^{u}$ cannot wind onto $\tilde{\gamma}$. Finally, $\tilde{v} \in \mathbf{T}$.

\subsubsection{Extension to the whole sphere}

Note that we described above only a part of the phase portrait of a vector field $v$ of class T. Such a vector field may be extended to the whole sphere in many different ways. One extension of a slightly perturbed vector field is shown in Figure 2, Let us describe an extension of $v$, beginning with the interior of the loop $l$. There are exactly three singular points inside this loop: the saddle $I$, one sink and one source. They are hyperbolic by Assumption 1. The outgoing separatrixes of the saddle $I$ tend to the sink. The closure of their union bounds a domain homeomorphic to a disc. There is one source in this domain; the interior of this domain is the repulsion basin of this source. The closure of this basin is a so called Cherry cell, see Figure 2 .

The phase portrait of $v$ outside the polycycle $\gamma$ is constructed in a similar way. The polycycle $\gamma$ is surrounded by a repelling hyperbolic limit cycle; two incoming separatrixes of $E$ tend to this cycle in the negative time; the domain bounded by these separatrixes is a Cherry cell, see Figure 2. In the simplest case, this limit cycle is the limit cycle near infinity. In other cases, the phase portrait outside this limit cycle may be more complicated; in particular, it may include other polycycles homeomorphic to $\gamma$.

The interior of the "heart" part of the polycycle $\gamma$ (union of connections $L M$ and $M L$ ) contains one sink, one source and no limit cycles. One outgoing separatrix of the saddle $M$ tends to the sink. One incoming separatrix of $M$ emerges from the source.

The extension described above is in a sense a key example. In what follows, we will describe only a part of the phase portraits of the vector fields considered, paying no attention to the extension of these parts to the whole sphere. These extensions are analogous to the example described above.

\subsection{Generic unfoldings of vector fields of class $\mathrm{T}$}

\subsubsection{Local families and unfoldings}

In what follows, we deal with local families. 


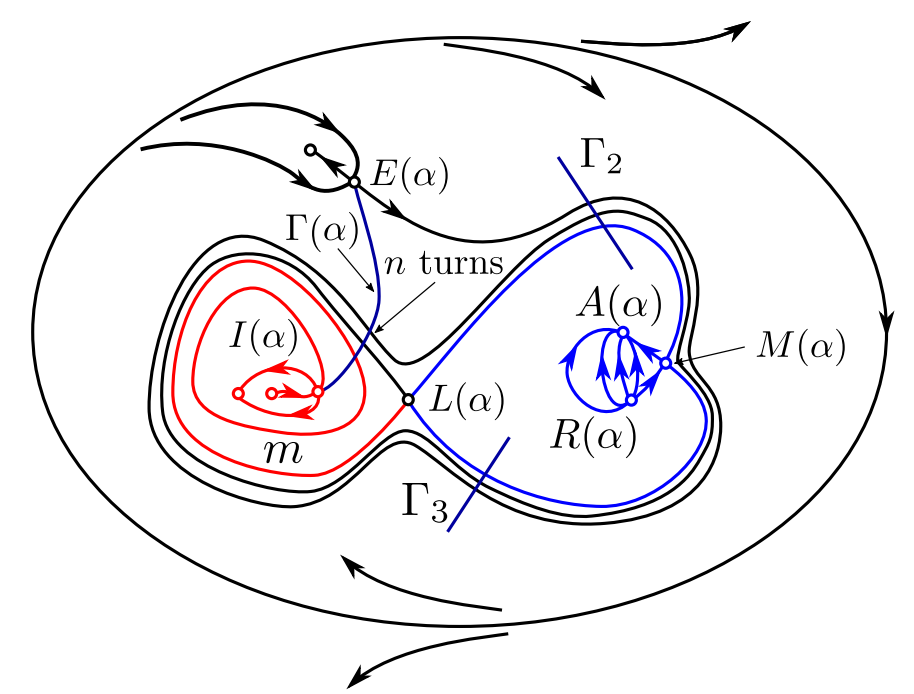

Figure 2: Sparkling saddle connections of an unfolding of $v$ and an extension of the phase portrait to the whole sphere

Definition 9. A local family at $\alpha=0$ with the base $\left(\mathbb{R}^{k}, 0\right)$ is a germ on $\{0\} \times M$ of a family given on $B \times M, B \ni 0, B$ is open. Two local families are moderately topologically equivalent if they have locally moderately topologically equivalent representatives, and the corresponding homeomorphism of the bases maps 0 to 0 .

Denote by $\mathcal{V}_{k}^{\text {loc }}\left(S^{2}\right)$ the set of local $k$-parameter families of $C^{3}$ smooth vector fields in $S^{2}$.

Definition 10. An unfolding of a vector field is a local family for which this field corresponds to the critical (zero) parameter value. We say that this family unfolds the vector field.

We will be mainly interested in generic unfoldings.

For a Banach submanifold $\mathbf{T} \subset \operatorname{Vect}\left(S^{2}\right)$, denote by $\mathbf{T}_{k}^{\pitchfork}$ the set of $k$-parameter unfoldings of vector fields $v \in \mathbf{T}$ transversal to $\mathbf{T}$ at $v$.

\subsubsection{Genericity assumptions}

Consider an unperturbed vector field $v \in \mathbf{T}$. Let $\gamma$ be the polycycle of the vector field $v$ of the type $T H$. If $v$ has several polycycles homeomorphic to $\gamma$, we fix any of them, say $\gamma_{j}$, and let $\gamma=\gamma_{j}$.

Fix cross-sections $\Gamma_{2}, \Gamma_{3}$ to the $\operatorname{arcs} L M$ and $M L$ of $\gamma$, respectively, and let $\Gamma_{1}=\Gamma$, see Figure 2, Let $O_{j}$ be the only intersection point of $\gamma$ with $\Gamma_{j}, O_{1}=O$. Fix coordinates $x_{j}: \Gamma_{j} \rightarrow \mathbb{R}$ that orient $\Gamma_{j}$ from inside to outside of $\gamma$.

Now consider a three parameter local family $V=\left\{v_{\alpha}\right\}$ that unfolds $v$. Let $\alpha \in\left(\mathbb{R}^{3}, 0\right)$ be the parameter, and $v_{\alpha}$ be the corresponding vector field of the family, $v=v_{0}$. For $\alpha$ close to zero, the vector field $v_{\alpha}$ has two saddles $L(\alpha)$ and $M(\alpha)$ smoothly depending on $\alpha$, together with their separatrixes, $L(0)=L, M(0)=M$. 
For the initial vector field, each point $O_{j}$ belongs to two separatrixes, one unstable and one stable. For $\alpha$ close to zero, let $U_{j}(\alpha)$ and $S_{j}(\alpha)$ be the first intersection points of the separatrixes of the saddles $L(\alpha)$ and $M(\alpha)$ with the cross-sections $\Gamma_{j} ; U$ corresponds to the unstable separatrixes, $S$ corresponds to the stable ones; $S_{j}(0)=U_{j}(0)=O_{j}$.

Let us introduce separatrix splitting parameters $\sigma_{j}$ well defined for small $\alpha$ :

$$
\sigma_{j}(\alpha)=x_{j}\left(S_{j}(\alpha)\right)-x_{j}\left(U_{j}(\alpha)\right) \text {. }
$$

We also introduce the separatrix splitting map,

$$
\sigma(\alpha)=\left(\sigma_{1}(\alpha), \sigma_{2}(\alpha), \sigma_{3}(\alpha)\right)
$$

The only genericity assumption on the unfolding of $v$ is

$$
\operatorname{det} \frac{\partial \sigma}{\partial \alpha}(0) \neq 0 \text {. }
$$

It is easy to check that this condition does not depend on the choice of $\Gamma_{j}$ and $x_{j}$. Relation (8) allows us to take $\sigma$ as a new parameter of the local family $V$.

Remark 2. Though $\Gamma_{1}=\Gamma$ depends on $\alpha$ (see Section 2.2.5 below), we may and will assume that the intersection $\Gamma_{1}^{\prime}$ of $\Gamma_{1}$ with a small neighborhood of $O$ does not depend on $\alpha$, hence we can choose $x_{1}: \Gamma_{1}^{\prime} \rightarrow \mathbb{R}$, and define $\sigma_{1}(\alpha)$ for $\alpha$ small enough. Local cross sections $\Gamma_{2}$ and $\Gamma_{3}$ do not depend on $\alpha$; hence, $\sigma_{2}(\alpha)$ and $\sigma_{3}(\alpha)$ are well defined.

\subsubsection{Class $\mathrm{T}$ and Banach submanifolds}

Let us prove that the class $\mathbf{T}$ is a Banach submanifold in some neighborhood of any point $v \in \stackrel{\circ}{\mathbf{T}}$. Such a vector field is a generic point in $\mathbf{T}$. By definition of a Banach submanifold, it is sufficient to find a map $\sigma:\left(\operatorname{Vect}\left(S^{2}\right), v\right) \rightarrow\left(\mathbb{R}^{3}, 0\right)$ such that $(\mathbf{T}, v)=\sigma^{-1}(0)$, and rank of $d \sigma(v)$ equals 3. The map $\sigma$ is given by (7). Since $v$ has no saddle connections except for the edges of $\gamma$, and all singular points of $v$ are hyperbolic, the equation $\sigma=0$ actually defines $\mathbf{T}$ near $v$. Next, the map $\sigma$ has a full rank since we may use a partition of unity to perturb one of $\sigma_{j}$ without touching the other two. Note that relation (8) is equivalent to $V \in \mathbf{T}_{3}^{\pitchfork}$.

Near the points $v$ corresponding to vector fields that have several polycycles homeomorphic to $\gamma$, say $\gamma_{1}, \ldots, \gamma_{D}$, the set $\mathbf{T}$ is no more a manifold. Let $v$ be such a field. Denote by $\mathbf{T}_{\gamma_{1}}$ the set of those vector fields $w \in \mathbf{T}$ close to $v$ that have a polycycle $\gamma_{1}(w)$ homeomorphic to $\gamma$, continuously depending on $w$ and such that $\gamma_{1}(v)=\gamma_{1}$. The set $\mathbf{T}_{\gamma_{1}}$ is a Banach manifold in some neighborhood of $v$. The set $\mathbf{T}$ near $v$ is a union of the manifolds $\mathbf{T}_{\gamma_{1}}, \ldots, \mathbf{T}_{\gamma_{D}}$, and has self-intersections.

\subsubsection{Sparkling saddle connections in a family of class $\mathrm{T}_{3}^{\pitchfork}$ : a heuristic description}

Let a vector field $v \in \mathbf{T}$ have exactly one polycycle $\gamma$ of the type $T H$. Let $V$ be a three-parameter unfolding of $v$ transversal to $\mathbf{T} ; \sigma=\left(\sigma_{1}, \sigma_{2}, \sigma_{3}\right)$ be the parameter of this family defined above. 
Consider the line $\mathcal{E}=\left\{\sigma_{2}=\sigma_{3}=0\right\}$ in the parameter space. It is parametrized by $\sigma_{1}$, and corresponds to vector fields $v_{\alpha}$ with the two connections between $L(\alpha)$ and $M(\alpha)$ unbroken. The parameter $\sigma_{1}$ is special; let us redenote it by $\varepsilon$.

Let us give a heuristic description of some bifurcations in the family $V$ that occur as the parameter $\alpha$ changes along $\mathcal{E}$. Let $I(\alpha), E(\alpha)$ be hyperbolic saddles of $v_{\alpha}$ smoothly depending on $\alpha, I(0)=I, E(0)=E$. There are two sequences of sparkling saddle connections:

exterior connection: the outgoing separatrix $W_{E}^{u}(\alpha)$ of the saddle $E(\alpha)$ makes $n$ turns (the precise meaning of this term is defined below) around the whole polycycle $\gamma$, then coincides with the incoming separatrix of the saddle $L(\alpha)$;

interior connection: the incoming separatrix $W_{I}^{s}(\alpha)$ of the saddle $I(\alpha)$ makes $m$ turns along $l$ in the backward time, then coincides with the outgoing separatrix of the saddle $L(\alpha)$.

These connections (that do not in general occur simultaneously) are shown in Figure 2. For simplicity, they are shown in one and the same figure.

\subsubsection{Sparkling saddle connections in a family of class $\mathrm{T}_{3}^{\dagger}$ : a detailed description}

Let us describe in more detail the bifurcations in the family $V$ that occur when the parameter changes along the line $\mathcal{E}$. Let $\Gamma(\alpha)$ be a smooth family of arcs connecting $I(\alpha)$ with $E(\alpha)$ and transversal to $v_{\alpha}, \Gamma(0)=\Gamma$.

Definition 11. We say that an exterior (resp., an interior) saddle connection $E(\alpha) L(\alpha)$ (resp., $L(\alpha) I(\alpha)$ ) makes $n$ turns around $\gamma$ (resp., $m$ turns around $l$ ) provided that it intersects the curve $\Gamma(\alpha)$ with endpoints excluded at exactly $n$ points (resp., $m$ points).

Remark 3. The number of turns is not an invariant of the orbital topological classification of vector fields on the two-sphere. Indeed, $n$ and $m$ depend not only on $v_{\alpha}$, but also on the choice of $\Gamma(\alpha)$.

We fix the family $\Gamma(\alpha)$, and thus speak about the number of turns.

The following lemma describes the asymptotic behavior of the corresponding values of the parameter $\varepsilon$ near zero.

Lemma 1 (Asymptotic Lemma). For $m$ and $n$ sufficiently large the following holds. There exists only one value $\varepsilon=i_{m}$ (respectively, $\varepsilon=e_{n}$ ) that corresponds to a vector field with an interior (respectively, an exterior) sparkling separatrix connection with $m$ (respectively, $n$ ) turns. The sequences $i_{m}$ and $e_{n}$ satisfy the asymptotic equalities

$$
\begin{aligned}
& \log \left(-\log i_{m}\right)=m \log \lambda^{-1}+O(1), \\
& \log \left(-\log e_{n}\right)=n \log \left(\lambda^{2} \mu\right)+O(1),
\end{aligned}
$$

and are monotonically decreasing. 
Remark 4 . The choice of the cross section $\Gamma$ and the family $\Gamma(\alpha)$ does not affect the statement of the lemma: another choice may change the number of turns by a constant term only.

Remark 5. In fact, for a sufficiently smooth family of vector fields, $O(1)$ in $(9)$ and $(10)$ can be replaced by $a+r_{m}$ and $b+\rho_{n}$, respectively, where $a$ and $b$ are constants and $r_{m}$, and $\rho_{n}$ tend to 0 exponentially. We do not need this fact for our analysis. Its proof requires more technical details, so we will skip it.

Lemma 1 is the most technical part of the proof. We postpone its proof to Section 4. Let us deduce the theorem from it.

\subsection{Two equivalent families of class $\mathbf{T}_{3}^{\dagger}$}

Begin with some notes about the real line.

\subsubsection{Topology of two sequences in the line}

Parameter values $e_{n}$ corresponding to exterior sparkling saddle connections and $i_{m}$ corresponding to interior ones form two decreasing sequences converging to 0 from above. As shown below, for two equivalent families of class $\mathbf{T}_{3}^{\dagger}$ there exists a homeomorphism $\left(\mathbb{R}_{+}, 0\right) \rightarrow\left(\mathbb{R}_{+}, 0\right)^{\prime}$ of neighborhoods of zero on the real line $\mathcal{E}$ parametrized by $\varepsilon$ that brings the couple of sequences $\left(i_{m}\right),\left(e_{n}\right)$ corresponding to one family to the couple of analogous sequences corresponding to another one.

Definition 12. Two couples of bounded countable sets $A, B$ and $\tilde{A}, \tilde{B}$ in $\mathbb{R}_{+}$with the only accumulation point zero are called equivalent provided that there exists a germ of a homeomorphism $h:\left(\mathbb{R}_{+}, 0\right) \rightarrow\left(\mathbb{R}_{+}, 0\right)$ that links the germs of the corresponding sets at zero:

$$
h(A, 0)=(\tilde{A}, 0), \quad h(B, 0)=(\tilde{B}, 0) .
$$

Equivalence classes of pairs thus defined may have a lot of topological invariants. We present here only one called relative density. Enumerate the elements of each of the countable sets in the definition above in a monotonic decreasing order. Let $N_{A}(x)$ be the counting function

$$
N_{A}(x)=\#\{a \in A \mid a \geq x\} .
$$

In the same way the counting function is defined for the other sets $B, \tilde{A}, \tilde{B}$. The relative density of two sets $A$ and $B$ is the limit (if exists):

$$
\nu(A, B)=\lim _{x \rightarrow 0} \frac{N_{A}(x)}{N_{B}(x)} .
$$

Clearly, $\nu(A, B)$ depends only on the germs of $A$ and $B$ at zero, and $\nu(A, B)=\nu(\tilde{A}, \tilde{B})$ provided that $(A, B)$ is equivalent to $(\tilde{A}, \tilde{B})$.

Lemma 1 motivates the following definition 
Definition 13. A set $A \subset \mathbb{R}$ is called a quasi (arithmetic) progression with the difference $\delta_{A}$ if its germ at infinity has the form

$$
(A,+\infty)=\left\{\delta_{A} k+O(1) \mid k \in \mathbb{N}\right\} .
$$

Lemma 2. Let $A, B \subset \mathbb{R}_{+}$be two countable bounded sets with the only accumulation point at zero. Suppose that there exists a homeomorphism $\mathcal{L}:\left(\mathbb{R}_{+}, 0\right) \rightarrow\left(\mathbb{R}_{+},+\infty\right)$ such that $\mathcal{L}(A)$ and $\mathcal{L}(B)$ are quasi progressions with differences $\delta_{A}$ and $\delta_{B}$, respectively. Then the relative density $\nu(A, B)$ exists and is given by

$$
\nu(A, B)=\frac{\delta_{B}}{\delta_{A}}
$$

The proof is obvious. This lemma together with Lemma 1 immediately implies the following statement.

Corollary 1. The sequences $\left(e_{n}\right),\left(i_{m}\right)$ introduced in Lemma 1 have the relative density

$$
\nu\left(\left(e_{n}\right),\left(i_{m}\right)\right)=\frac{-\log \lambda}{\log \lambda^{2} \mu} .
$$

\subsubsection{A topologically distinguished one-parameter subfamily}

Let $V$ and $\mathcal{E}$ be the same as in Section 2.2.4. We will describe $\mathcal{E}$ in geometric terms.

Proposition 2. Let $L$ and $M$ be two hyperbolic saddles of a $C^{1}$-smooth vector field $v_{0}$ on $S^{2}$. Suppose that $v_{0}$ has a saddle connection $l$ between $L$ and $M$. Let $\sigma_{l}$ be a separatrix splitting parameter $v \mapsto \sigma_{l}(v)$ defined for the vector fields $C^{1}$-close to $v_{0}$ as it was done in Section 2.2.2, see (6). Then there exists a neighborhood $U$ of $l$ in $S^{2}$ and a neighborhood $W$ of $v_{0}$ in $\operatorname{Vect}\left(S^{2}\right)$ such that if a vector field $v \in U$ has a saddle connection that belongs to $U$ then $\sigma_{l}(v)=0$. The proposition holds true if we replace $\operatorname{Vect}\left(S^{2}\right)$ by a family that unfolds $v_{0}$, and $W$ is a neighborhood of 0 in the base of the family.

The proof is trivial. Yet the proposition becomes wrong if we omit the requirement that the connection between the saddles $L(v)$ and $M(v)$ close to $L$ and $M$ is close to the original connection itself. This happens due to sparkling saddle connections.

By Proposition 2, the family $\mathcal{E}$ is characterized by the following condition: for $\sigma$ small, the vector field $v_{\sigma}$ has a polycycle "heart" with the edges close to the saddle connections $L M$ and $M L$ of the vector field $v_{0}$ if and only if $\sigma_{2}=\sigma_{3}=0$, that is, $\sigma \in \mathcal{E}$.

\subsubsection{Numeric invariants of families of class $\mathrm{T}_{3}^{\pitchfork}$}

Let $V$ and $\tilde{V}$ be two families as in Theorem 4. Let $H=\left(h, H_{\alpha}\right)$ be a moderate topological equivalence (1) that links the families $V$ and $\tilde{V}, h$ be the corresponding map of the parameter spaces. Let us shift coordinates in the bases of $V$ and $\tilde{V}$ so that $v_{0}, \tilde{v}_{0}$ are unique points of $V \cap \mathbf{T}$ and $\tilde{V} \cap \mathbf{T}$, respectively. Proposition 1 implies that $h(0)=0$. 
In this subsection, redenote by $V, \tilde{V}$ the local families $(V, v),(\tilde{V}, \tilde{v})$ parametrized by $\sigma$ and $\tilde{\sigma}$. Let $\tilde{\mathcal{E}}$ be the local subfamily of $\tilde{V}$, analogouse to $\mathcal{E} \subset V$, i.e. given by equations $\tilde{\sigma}_{2}=\tilde{\sigma}_{3}=0$. We want to prove that $h(\mathcal{E})=\tilde{\mathcal{E}}$.

Let $\gamma$ be the polycycle of $v_{0}$ of the type $T H, L$ and $M$ be its vertexes. Due to Proposition 1, $\tilde{\gamma}=H_{0}(\gamma)$ is a hyperbolic polycycle of $\tilde{v}_{0}$ with vertexes $\tilde{L}=H_{0}(L)$, $M=H_{0}(M)$. Let $U$ and $W$ be the neighborhoods of $\tilde{L} \tilde{M}$ in $S^{2}$ and 0 in $\mathbb{R}^{3}$ from Proposition 2. If $\sigma_{2}=0$, then the vector field $v_{\sigma}$ has a saddle connection $L(\sigma) M(\sigma)$ close to $L M$. As $H$ is moderate, the saddle connections $\tilde{L}(\sigma) \tilde{M}(\sigma)=H_{\sigma}(L(\sigma) M(\sigma))$ belong to $U$ for $\sigma$ small, and $h(\sigma)$ belongs to $W$. By Proposition 2, $\tilde{\sigma}_{2}(h(\sigma))=0$. Same arguments imply that if $\sigma_{3}(\sigma)=0$ then $\tilde{\sigma}_{3}(h(\sigma))=0$ for $\sigma$ small. Hence, $h(\mathcal{E})=\tilde{\mathcal{E}}$.

Let $\varepsilon=\sigma_{1}, \tilde{\varepsilon}=\tilde{\sigma}_{1}$ be the charts on $\mathcal{E}, \tilde{\mathcal{E}}$ respectively, and $\psi:(\mathbb{R}, 0) \rightarrow(\mathbb{R}, 0)$ be the restriction $\left.h\right|_{\mathcal{E}}$ written in the charts $\varepsilon$, $\tilde{\varepsilon}$. It is a homeomorphism. Let $\tilde{E}=H_{0}(E)$. By Proposition 1, $\tilde{E}$ is the unique saddle whose unstable separatrix winds towards the polycycle $\tilde{\gamma}$. Let $\left(\tilde{e}_{n}\right)$ be the sequence of the parameter values $\tilde{\varepsilon}$ for which the vector field $\tilde{v}_{\sigma}$ has a sparkling saddle connection between $\tilde{E}(\tilde{\sigma})$ and $\tilde{L}(\tilde{\sigma})$ for $\tilde{\sigma}=(\tilde{\varepsilon}, 0,0)$. The phase portraits of the vector fields $v_{\sigma}$ and $\tilde{v}_{\tilde{\sigma}}, \tilde{\sigma}=h(\sigma)$, are linked by a homeomorphism. Hence, they simultaneously have or have not sparkling saddle connections between $E(\sigma)$, $L(\sigma)$ and $\tilde{E}(\tilde{\sigma}), \tilde{L}(\tilde{\sigma})$. Thus $\psi$ sends the sequence $\left(e_{n}\right)$ to the sequence $\left(\tilde{e}_{n}\right)$, possibly shifting the numeration. In the same way, the sequence $\left(\tilde{i}_{m}\right)$ is defined, and the relation $\psi\left(i_{m}\right)=\tilde{i}_{m-a}$ for some $a$ is proved.

Due to Corollary 1, the relative densities $\nu\left(\left(e_{n}\right),\left(i_{m}\right)\right)$ and $\nu\left(\left(\tilde{e}_{n}\right),\left(\tilde{i}_{m}\right)\right)$ exist and are given by (11). Since the pairs of sequences $\left(\left(e_{n}\right),\left(i_{m}\right)\right)$ and $\left(\left(\tilde{e}_{n}\right),\left(\tilde{i}_{m}\right)\right)$ are equivalent in sense of Definition 12, their relative densities coincide. Hence,

$$
\frac{\log \lambda^{-1}}{\log \lambda^{2} \mu}=\frac{\log \tilde{\lambda}^{-1}}{\log \tilde{\lambda}^{2} \tilde{\mu}} .
$$

Theorem 4, modulo Lemma 1, is proved.

\section{Functional invariants}

In this section we construct an open set of families having functional invariants; in particular, we prove Theorems 2 and 3 , modulo Lemma 3 stated below. This lemma is an analog of Lemma 1. It is proved in Section 4.

\subsection{Heuristic description of functional invariants}

Consider the class $\mathbf{T}$ described in Section 2.1. Choose a vector field $v$ of this class, and a polycycle $\gamma$ of $v$ of the class $T H$; if it is not unique, we fix one arbitrary. For vector fields $w$ close to $v$ on $\mathbf{T}_{\gamma}$, the following objects are defined:

- two saddles $L(w)$ and $M(w)$;

- their characteristic numbers $\lambda(w)$ and $\mu(w)$; 
- the function $\nu_{\gamma}:\left(\mathbf{T}_{\gamma}, v\right) \rightarrow\left(\mathbb{R}_{+}, \nu_{\gamma}(v)\right)$ given by

$$
\nu_{\gamma}(w)=\frac{-\log \lambda(w)}{\log \lambda^{2}(w) \mu(w)},
$$

cf. (5).

Class $\mathbf{T}_{\gamma}$ has codimension 3. Consider the class $\left(\mathbf{T}_{\gamma}\right)_{4}^{\pitchfork}$, see Section 2.2.1. A family $V$ of class $\left(\mathbf{T}_{\gamma}\right)_{4}^{\pitchfork}$ intersects the class $\mathbf{T}_{\gamma}$ at a one-parameter family $J_{V}$. For a generic family $V \in\left(\mathbf{T}_{\gamma}\right)_{4}^{\dagger}$, the function $\left.\nu\right|_{J_{V}}$ is a natural parameter on $J_{V}$.

Consider now a class $\mathbf{T}^{2} \subset \mathbf{T}$ of vector fields that contain two polycycles $\gamma_{1}, \gamma_{2}$ of the type $T H$. Let $\mathbf{T}_{\gamma_{1}} \subset \mathbf{T}$ and $\mathbf{T}_{\gamma_{2}} \subset \mathbf{T}$ be Banach manifolds described in Section 2.2.3. Then $\mathbf{T}^{2}=\mathbf{T}_{\gamma_{1}} \cap \mathbf{T}_{\gamma_{2}} \subset \mathbf{T}$.

As it was mentioned above, the objects $L_{j}(v), M_{j}(v), \lambda_{j}(v), \mu_{j}(v)$ and $\nu_{j}(v)$ are well defined on the manifold $\mathbf{T}_{\gamma_{j}}, j=1,2$. The class $\mathbf{T}^{2}$ has codimension six. There are two functions $\nu_{j}=\nu_{\gamma_{j}}$ defined on the manifolds $\mathbf{T}_{\gamma_{j}}$.

Consider the class $\left(\mathbf{T}^{2}\right)_{7}^{\pitchfork}$. A family $V \in\left(\mathbf{T}^{2}\right)_{7}^{\dagger}$ again intersects the class $\mathbf{T}^{2}$ by a oneparameter family $J_{V}$. Now, there are two natural parameters on $J_{V}$ : restrictions to $J_{V}$ of the functions $\nu_{1}$ and $\nu_{2}$. One natural parameter is a function of another one. This is a functional invariant of the moderate topological classification of families of class $\left(\mathbf{T}^{2}\right)_{7}^{\mathrm{h}}$.

In a similar way, increasing the order of degeneracy, one can construct classes of vector fields with $D$ functions like $\nu(v)$, and consider families that intersect this class by $d$-dimensional subfamilies, thus obtaining functional invariant of the form of a germ of a $\operatorname{map}\left(\mathbb{R}_{+}^{d}, a\right) \rightarrow\left(\mathbb{R}_{+}^{d^{\prime}}, b\right), d^{\prime}=D-d, a \in \mathbb{R}_{+}^{d}, b \in \mathbb{R}_{+}^{d^{\prime}}$.

The latter conclusion needs to be justified. We pass to the rigorous presentation and start with a notion of a function invariant under the moderate topological classification of local families. In what follows, we construct six (not seven) parameter families with functional invariants; the constructions are a little bit more tricky than above. Notation $\mathbf{T}^{2}$ will not be used any more.

\subsection{Families with invariant functions: definition}

Let $d, d^{\prime}$ be arbitrary natural numbers; later on they will be the same as in Theorem 3 . Put $D=d+d^{\prime}$. As in Section 2, we shall construct a Banach submanifold $\mathbf{T}_{D}$, then consider the class $\left(\mathbf{T}_{D}\right)_{k}^{m}$ of local $k$-parameter families transversal to this submanifold. The main difference with Section 2 is that now the codimension of $\mathbf{T}_{D}$ is less than the number of parameters in the family,

$$
\operatorname{codim} \mathbf{T}_{D}=2 D+1<2 D+1+d=k .
$$

Therefore, each family $V$ with $k>$ codim $\mathbf{T}_{D}$ includes a subfamily $J_{V}=V \cap \mathbf{T}_{D}$ with $k-\operatorname{codim} \mathbf{T}_{D}=d$ parameters.

Definition 14. Let $T$ be a Banach submanifold in some open subset of $\operatorname{Vect}\left(S^{2}\right), k$ be an integer number, $k \geq \operatorname{codim} T$. A function $\varphi: T \rightarrow \mathbb{R}$ is an invariant function of 
the moderate topological classification of families of class $T_{k}^{\dagger}$ provided that the following holds. Let two families $V, \tilde{V} \in T_{k}^{\dagger}$ with the bases $B, \tilde{B}$ be moderately topologically equivalent. Consider the subfamilies $J_{V}=V \cap T, J_{\tilde{V}}=\tilde{V} \cap T$ with the bases $B\left(J_{V}\right) \subset B$, $B\left(J_{\tilde{V}}\right) \subset \tilde{B}$, respectively. Then

$$
h\left(B\left(J_{V}\right)\right)=B\left(J_{\tilde{V}}\right), \quad \varphi\left(v_{\alpha}\right)=\varphi\left(\tilde{v}_{h(\alpha)}\right) \quad \text { for } v_{\alpha} \in J_{V} .
$$

The first relation means that $T$ is topologically distinguished in its neighborhood. The second relation means that $\varphi$ takes the same values on the corresponding vector fields of the equivalent families.

Denote by $\varphi_{V}: B\left(J_{V}\right) \rightarrow \mathbb{R}$ the function $\left.\varphi\right|_{J_{V}}$ written in the chart $\alpha, \varphi_{V}(\alpha)=\varphi\left(v_{\alpha}\right)$; denote by $\chi$ the restriction $\left.h\right|_{J_{V}}$ written in the charts $\alpha, \tilde{\alpha}$. Then 12 takes the form

$$
\varphi_{\tilde{V}} \circ \chi=\varphi_{V} .
$$

We shall use this form later.

Remark 6 . The notion of an invariant function $T \rightarrow \mathbb{R}$ should not be confused with the notion of a functional invariant. If $k=\operatorname{codim} T$, then $J_{V}$ consists of one point, and an invariant function provides a numeric invariant.

\subsection{Factory of invariant functions}

\subsubsection{A special polycycle of higher codimension}

Consider a normalized vector field $v_{0}$ with a hyperbolic polycycle $\gamma_{e}^{0}$ monodromic from the exterior. Suppose that $\gamma_{e}^{0}$ includes a polycycle $\gamma_{i}^{0}$ monodromic from the interior, see Figure 3.

In order to introduce a condition analogous to (2), we need the following definition.

Definition 15. The characteristic number of a hyperbolic polycycle $\gamma$ is the product of the characteristic numbers of all the saddles met during one turn along the polycycle; it is denoted by $\lambda(\gamma)$.

Suppose that

$$
\lambda\left(\gamma_{e}^{0}\right)>1, \quad \lambda\left(\gamma_{i}^{0}\right)<1
$$

Example 1. The characteristic numbers of the polycycles $\gamma$ and $l$ from Section 2 are equal to $\lambda^{2} \mu$ and $\lambda$, respectively. So, in this case (14) becomes (2).

Polycycles $\gamma_{e}^{0}, \gamma_{i}^{0}$ will be called special for future references.

\subsubsection{Class $\hat{\mathrm{T}}$}

Let us now construct a class $\hat{\mathbf{T}}=\hat{\mathbf{T}}\left(\gamma_{e}^{0}, \gamma_{i}^{0}\right)$. Vector fields $v \in \hat{\mathbf{T}}$ are required to have polycycles $\gamma_{i}(v) \subset \gamma_{e}(v)$ such that the couple $\left(\gamma_{e}, \gamma_{i}\right)$ is homeomorphic to $\left(\gamma_{e}^{0}, \gamma_{i}^{0}\right)$. At this spot, for the abuse of notation, we skip the expression $(v)$ that indicates the dependence 
of different objects on $v$. Assume also that $v$ has no saddle connections other than the edges of $\gamma_{e}$.

Moreover, let $E$ and $I$ be hyperbolic saddles outside $\gamma_{e}$ and inside $\gamma_{i}$ respectively. Suppose that there exists a smooth arc $\Gamma$ that connects $E$ and $I$ and is transversal to $v$ in its interior points; let $O$ be the unique intersection point $\Gamma \cap \gamma_{i}$, and $l \subset \gamma_{i}$ be the saddle connection, edge of $\gamma_{i}$, that contains $O$. Denote by $\Gamma^{+}$and $\Gamma^{-}$half open arcs of $\Gamma$ between $O$ and $E$ ( $E$ excluded), and between $O$ and $I$ ( $I$ excluded) respectively. Note that the germs of the monodromy maps

$$
\Delta_{e}:\left(\Gamma^{+}, O\right) \rightarrow\left(\Gamma^{+}, O\right), \quad \Delta_{i}:\left(\Gamma^{-}, O\right) \rightarrow\left(\Gamma^{-}, O\right)
$$

along the polycycles $\gamma_{e}, \gamma_{i}$ are well defined. Suppose that the germs $\Delta_{e}, \Delta_{i}^{-1}$ may be extended as global Poincaré maps of the vector field $v$ :

$$
\Delta_{e}: \Gamma^{+} \rightarrow \Gamma^{+}, \quad \Delta_{i}^{-1}: \Gamma^{-} \rightarrow \Gamma^{-}
$$

that have no fixed points except for $O$; both maps are into. Then $O$ is an attracting fixed point of $\Delta_{e}$ and of $\Delta_{i}^{-1}$; this follows from (14), see Remark 12 below.

Let $\hat{\mathcal{H}}$, cf. Section 2.1.3, be the class of vector fields $v$ such that

- all the singular points and polycycles of the vector field $v$ are hyperbolic;

- products of all the characteristic values of the saddles of $v$ taken in the powers 0 , 1,2 , where at least one power is non-zero, are different from 1.

Clearly, $\hat{\mathcal{H}}$ is open and dense in $\operatorname{Vect}\left(S^{2}\right)$. We assume that $v \in \hat{\mathcal{H}}$. We also assume that the following analog of Assumption 1 from Section 2.1.3 holds for the class $\hat{\mathbf{T}}$.

Assumption 2. For any polycycle $\gamma$ of a vector fields $v \in \hat{\mathbf{T}}$ there is no more than one saddle whose separatrix winds toward $\gamma$ in the positive or negative time.

This completes the definition of the class $\hat{\mathbf{T}}$.

Proposition 3 (cf. Proposition 1). Consider two orbitally topologically equivalent vector fields $v, \tilde{v} \in \hat{\mathcal{H}}$. If $v \in \mathbf{T}$, then $\tilde{v} \in \hat{\mathbf{T}}$. Moreover, a homeomorphism provided by Definition 4 sends the polycycles $\gamma_{e}(v), \gamma_{i}(v)$, and the saddles $E(v), I(v)$ to similar objects for $\tilde{v}$.

Proof. Let $H$ be a homeomorphism that links the phase portraits of $v$ and $\tilde{v}$. Similarly to the proof of Proposition 1, the polycycles $\gamma_{e}(\tilde{v})=H\left(\gamma_{e}(v)\right), \gamma_{i}(\tilde{v})=H\left(\gamma_{i}(v)\right)$ and the saddles $\tilde{E}=H(E), I=H(I)$ satisfy all the requirements of the class $\hat{\mathbf{T}}$, possibly except for inequalities (14). Let us prove these inequalities.

Suppose that $\lambda\left(\gamma_{e}(\tilde{v})\right) \leq 1$. Note that each saddle is met at most twice during one turn along $\gamma_{e}(\tilde{v})$, hence $\lambda\left(\gamma_{e}(\tilde{v})\right)$ is a product of characteristic numbers of saddles of $\tilde{v}$ taken in the powers 0, 1, 2. By definition of $\hat{\mathcal{H}}$, this implies $\lambda\left(\gamma_{e}(\tilde{v})\right) \neq 1$, hence $\lambda\left(\gamma_{e}(\tilde{v})\right)<1$. By Remark 12, this contradicts the property of the saddle $E$, namely, that a separatrix of $E$ winds towards $\gamma_{e}(\tilde{v})$, and proves the left inequality in (14). One can prove the right inequality in (14) in the same way. 


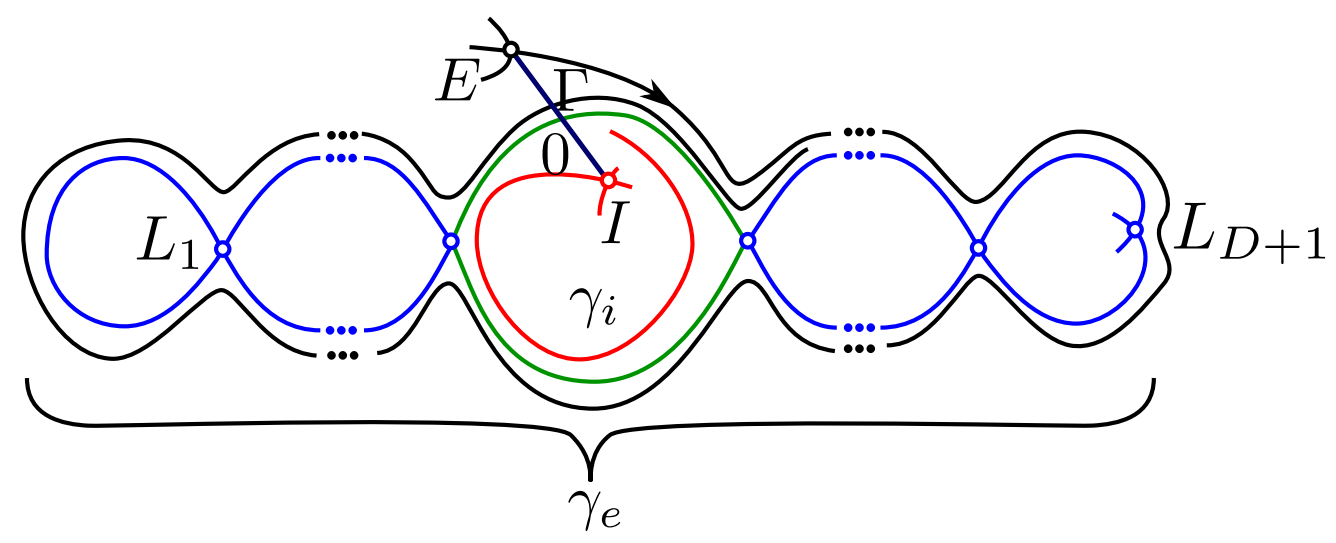

Figure 3: Factory of invariant functions

Let $\varphi$ be the function given by

$$
\varphi(v)=-\frac{\log \lambda\left(\gamma_{i}(v)\right)}{\log \lambda\left(\gamma_{e}(v)\right)}, \quad \varphi: \hat{\mathbf{T}} \rightarrow \mathbb{R}_{+}
$$

Fix $k \geq \operatorname{codim} \hat{\mathbf{T}}$. Consider the class $\hat{\mathbf{T}}_{k}^{\dagger}$. Recall that according to Section 2.2.1 this is the class of $k$-parameter unfoldings of vector fields of class $\hat{\mathbf{T}}$.

Theorem 5. The function $\varphi$ is invariant for the families of class $\hat{\mathbf{T}}_{k}^{\dagger}$ in sense of Definition 14.

Proof. We will prove Theorem 5 in the current Section 3.3. The proof follows the same lines as for Theorem 4 .

\subsubsection{Some bifurcations in the families of class $\hat{\mathbf{T}}_{k}^{\dagger}$}

Consider a local family $V=\left\{v_{\alpha} \mid \alpha \in\left(\mathbb{R}^{k}, 0\right)\right\}$ of class $\hat{\mathbf{T}}_{k}^{\dagger}$. By definition, $v_{0} \in \hat{\mathbf{T}}$. Let $\gamma_{e}, \gamma_{i}$ be two polycycles and let $l$ be a saddle connection of $v_{0}$ described in the previous subsection. As in Section 3.2, put $J_{V}=\hat{\mathbf{T}} \cap V, d:=\operatorname{dim} J_{V}$.

Let $l_{j}, j=1, \ldots, N$, be the edges of $\gamma_{e}, l=l_{1}$. Let $\sigma_{j}$ be the corresponding separatrix splitting parameters defined as in Section 2.2.2. Assume that the cross section $\Gamma_{1}$ used to define $\sigma_{1}$ coincides with $\Gamma$ near $\Gamma \cap l$. Put $\varepsilon=\sigma_{1}$.

Let $\mathcal{E}=\mathcal{E}_{V, l}$ be the $(d+1)$-dimensional subfamily of $V$ defined by

$$
\mathcal{E}_{V, l}=V \cap\left\{\sigma_{2}=\cdots=\sigma_{N}=0\right\} .
$$

Obviously, $J_{V} \subset \mathcal{E}$.

Let $\eta \in\left(\mathbb{R}^{d}, 0\right)$ be a coordinate on $B\left(J_{V}\right)$ smoothly extended to $B(\mathcal{E}), \eta\left(v_{0}\right)=0$. By the transversality of $V$ to $\hat{\mathbf{T}}, B(\mathcal{E})$ is a germ of a smooth submanifold of $B(V)$, and $\alpha=0$ is a non-critical point of the function $\varepsilon$ on $B(\mathcal{E})$. Then $\beta:=(\eta, \varepsilon)$ may be taken as a coordinate (new parameter) on $B(\mathcal{E})$ near 0 . By definition of $\varepsilon, J_{V}=$ $\left\{v_{\alpha} \mid v_{\alpha} \in \mathcal{E}, \varepsilon(\alpha)=0\right\}$. 
Vector fields $v_{\beta} \in \mathcal{E}$ have sparkling saddle connections between the saddles $E(\beta)$, $M(\beta)$ on one hand, and $L(\beta), I(\beta)$ on the other hand (exterior and interior connections respectively). Let $\Gamma(\beta)$ be a cross section that plays for $v_{\beta}$ the same role as $\Gamma$ plays for $v=v_{0}$. Namely, $\Gamma(\beta)$ connects $E(\beta)$ and $I(\beta)$, and is transversal to $v_{\beta}$ at its interior points. Moreover, $\Gamma(\beta)$ continuously depends on $\beta$, and $\Gamma(0)=\Gamma$. We may assume that $\Gamma(\beta)$ coincides with $\Gamma$ outside some small neighborhoods of $E$ and $I$.

Definition 16 (cf. Definition 11). A connection $E(\beta) M(\beta)$ (respectively, $L(\beta) I(\beta)$ ) makes $n$ (respectively, $m$ ) turns around $\gamma_{e}$ (around $\gamma_{i}$ ) provided that it intersects the $\operatorname{arc} \Gamma(\beta)$ with endpoints excluded at exactly $n$ points (resp., $m$ points).

The following analog of Lemma 1 holds:

Lemma 3 (Generalized Asymptotic Lemma). Consider the family $\mathcal{E}=\left\{v_{\beta}\right\}$ described above, $\beta=(\eta, \varepsilon)$. For $m$, $n$ sufficiently large, the following holds. In some neighborhood of 0 in $\mathbb{R}^{d}$, non depending on $m$ and $n$, there exist uniquely determined positive functions $\varepsilon=i_{m}(\eta), \varepsilon=e_{n}(\eta)$ such that the vector field $v_{\beta}, \beta=\left(\eta, i_{m}(\eta)\right)\left(\beta=\left(\eta, e_{n}(\eta)\right)\right)$ has an interior (exterior) saddle connection that makes $m$ (respectively $n$ ) turns around $\gamma_{i}$ $\left(\right.$ around $\left.\gamma_{e}\right)$. The functional sequences $\left(i_{m}\right),\left(e_{n}\right)$ decrease monotonically and satisfy the equations:

$$
\begin{aligned}
& \log \left(-\log i_{m}(\eta)\right)=-m \log \lambda_{i}(\eta)+O(1), \\
& \log \left(-\log e_{n}(\eta)\right)=n \log \lambda_{e}(\eta)+O(1)
\end{aligned}
$$

where $\lambda_{i}(\eta)=\lambda\left(\gamma_{i}\left(v_{\beta}\right)\right), \lambda_{e}(\eta)=\lambda\left(\gamma_{e}\left(v_{\beta}\right)\right), \beta=(\eta, 0)$, and the remainder terms are uniformly bounded in $\eta \in\left(\mathbb{R}^{d}, 0\right)$.

Remark 7 . When $k=\operatorname{codim} \hat{\mathbf{T}}$, the base of $J_{V}$ is one point $\{0\}$, and functions $i_{m}, e_{n}$ become numbers. In this case Lemma 3 implies Lemma 1, setting $\hat{\mathbf{T}}=\mathbf{T}, k=3$.

This lemma is proved in Section 4 together with Lemma 1.

\subsubsection{Two equivalent families of class $\hat{\mathbf{T}}_{k}^{\dagger}$}

In what follows, we suppose that $k>\operatorname{codim} \hat{\mathbf{T}}$. If $k=\operatorname{codim} \hat{\mathbf{T}}$, then Theorem 5 is proved in the same way as Theorem 4. Consider two moderately topologically equivalent local families of class $\hat{\mathbf{T}}_{k}^{\mathrm{h}}: V=\left\{v_{\alpha}\right\}$ and $\widetilde{V}=\left\{\tilde{v}_{\tilde{\alpha}}\right\}$. Let $J_{V}=V \cap \hat{\mathbf{T}}, J_{\tilde{V}}=\tilde{V} \cap \hat{\mathbf{T}}$. Let $H=\left(h, H_{\alpha}\right), h(0)=0$, be a moderate equivalence (1) that links these two families. By Proposition 3, the class $\hat{\mathbf{T}}$ is topologically distinguished in its neighborhood, hence $h\left(B\left(J_{V}\right)\right)=B\left(J_{\tilde{V}}\right)$.

Let $l=l_{1}, l_{j}$ for $j=2, \ldots, N$, and $\mathcal{E}$ be the same as in the previous subsection. Put $\tilde{l}=H_{0}(l), \tilde{l}_{j}=H_{0}\left(l_{j}\right)$. Let $\tilde{\sigma}_{j}: B(\tilde{V}) \rightarrow \mathbb{R}$ be the separatrix splitting parameters for $\tilde{l}_{j}, \tilde{\varepsilon}=\tilde{\sigma}_{1}$. Let $\tilde{\mathcal{E}}=\tilde{V} \cap\left\{\tilde{\sigma}_{2}=\cdots=\tilde{\sigma}_{N}=0\right\}$. Similarly to Section 2.3.3. Proposition 2 implies that $h$ sends $\left\{\sigma_{j}=0\right\}$ to $\left\{\tilde{\sigma}_{j}=0\right\}$, hence $h$ sends $\mathcal{E}$ to $\mathcal{E}$.

Let $i_{m}$ and $e_{n}$ be the functions corresponding to sparkling saddle connections in the family $\mathcal{E}$ introduced in Lemma 3. Denote by $\mathcal{I}_{m} \subset \mathcal{E}, \mathcal{E}_{n} \subset \mathcal{E}$ the graphs of the functions $i_{m}, e_{n}: B\left(J_{V}\right) \rightarrow \mathbb{R}_{+}$, respectively, defined for $r$ small, and $m, n$ large enough, and by 
$\mathcal{I}_{m}^{+}$and $\mathcal{E}_{n}^{+}$the domains $\varepsilon>i_{m}(\eta), \varepsilon>e_{n}(\eta)$. Let $\widetilde{\mathcal{I}}_{m}, \widetilde{\mathcal{E}}_{n}, \widetilde{\mathcal{I}}_{m}^{+}, \widetilde{\mathcal{E}}_{n}^{+}$be the similar objects for $\widetilde{V}$.

The functional sequences $\left(i_{m}\right)$ and $\left(e_{n}\right)$ are monotonically decreasing by Lemma 3 . The sequence $\left(i_{m}\right)$ corresponds to saddle connections between the saddles $L(\alpha), I(\alpha)$, where $\alpha \in B(\mathcal{E}), \varepsilon(\alpha)=i_{m}(\eta(\alpha))$. We ignore here the fact that this connection makes $m$ turns. The vector field $v_{\alpha} \in \mathcal{E}$ that has a saddle connection $L(\alpha) I(\alpha)$ corresponds to a vector field $\tilde{v}_{\alpha} \in \widetilde{\mathcal{E}}$ that has a saddle connection $\tilde{L}(\tilde{\alpha}) \tilde{I}(\tilde{\alpha})$. Hence, the map $h$ brings the germ of the union of graphs $\bigcup_{m \geq m_{0}} \mathcal{I}_{m}$ at 0 to the germ of the union of graphs $\bigcup_{\tilde{m} \geq \tilde{m}_{0}}^{\infty} \widetilde{\mathcal{I}}_{\tilde{m}}$ at 0 . A similar statement holds fo the unions $\bigcup_{n \geq n_{0}} \mathcal{E}_{n}$ and $\bigcup_{\tilde{n} \geq \tilde{n}_{0}} \tilde{\mathcal{E}}_{\tilde{n}}$.

The sequences of domains $\left(\mathcal{I}_{m}^{+}\right),\left(\mathcal{E}_{n}^{+}\right),\left(\widetilde{\mathcal{I}}_{m}^{+}\right),\left(\widetilde{\mathcal{E}}_{n}^{+}\right)$are monotonically increasing with respect to inclusion. The homeomorphism $h$ respects this property. Hence, there exist integer $a$ and $b$ such that

$$
h\left(\mathcal{I}_{m}\right)=\left(\widetilde{\mathcal{I}}_{m-a}\right), \quad h\left(\mathcal{E}_{m}\right)=\left(\widetilde{\mathcal{E}}_{m-b}\right) .
$$

\subsubsection{Relative density of two functional sequences}

Simlarly to Section 2.3.1, we introduce the counting functions

$$
N_{i}(\eta, \varepsilon)=\#\left\{m>m_{0} \mid \varepsilon \leq i_{m}(\eta)\right\}, N_{e}(\eta, \varepsilon)=\#\left\{n>n_{0} \mid \varepsilon \leq e_{n}(\eta)\right\} .
$$

In other words, $N_{i}(\eta, \varepsilon)=m$ for $\beta=(\eta, \varepsilon) \in \mathcal{I}_{m+m_{0}+1} \backslash \mathcal{I}_{m+m_{0}}$, and similarly for $N_{e}$. In the same way, functions $\tilde{N}_{i}$ and $\tilde{N}_{e}$ are defined.

Define the relative density of $\left(i_{m}\right)$ and $\left(e_{n}\right)$ as a limit

$$
\nu(\eta)=\lim _{\substack{\left(\eta^{\prime}, \varepsilon\right) \rightarrow(\eta, 0) \\ \varepsilon>0}} \frac{N_{e}\left(\eta^{\prime}, \varepsilon\right)}{N_{i}\left(\eta^{\prime}, \varepsilon\right)}
$$

if exists. Then Lemma 3 implies that

$$
\begin{aligned}
\nu(\eta)=\lim _{\substack{\left(\eta^{\prime}, \varepsilon\right) \rightarrow(\eta, 0) \\
\varepsilon>0}} \frac{N_{e}\left(\eta^{\prime}, \varepsilon\right)}{N_{i}\left(\eta^{\prime}, \varepsilon\right)} & =\lim _{\substack{\left(\eta^{\prime}, \varepsilon\right) \rightarrow(\eta, 0) \\
\varepsilon>0}} \frac{\log (-\log \varepsilon)+O(1)}{\log \lambda_{e}\left(\eta^{\prime}\right)} \times \frac{-\log \lambda_{i}\left(\eta^{\prime}\right)}{\log (-\log \varepsilon)+O(1)} \\
& =-\frac{\log \lambda_{i}(\eta)}{\log \lambda_{e}(\eta)}=\varphi\left(v_{\eta, 0}\right)
\end{aligned}
$$

and a similar equality holds for $\tilde{N}_{e}$ and $\tilde{N}_{i}$.

\subsubsection{Invariance of the function $\varphi$}

We will deduce invariance of the function $\varphi$, see (16), from the properties (20) and (21). Consider a point $(\eta, 0) \in\left(\mathbb{R}^{d}, 0\right)$ and the corresponding point $(\tilde{\eta}, 0)=h(\eta, 0) \in\left(\mathbb{R}^{d}, 0\right)$. Let us compute the limit $\lim _{\substack{(\eta, \varepsilon) \rightarrow\left(\eta_{0}, 0\right) \\ \varepsilon>0}} \frac{\tilde{N}_{e}(h(\eta, \varepsilon))}{\tilde{N}_{i}(h(\eta, \varepsilon))}$ in two ways. On one hand, due to (20) 
and (21),

$$
\lim _{\substack{\left(\eta^{\prime}, \varepsilon\right) \rightarrow(\eta, 0) \\ \varepsilon>0}} \frac{\tilde{N}_{e}\left(h\left(\eta^{\prime}, \varepsilon\right)\right)}{\tilde{N}_{i}\left(h\left(\eta^{\prime}, \varepsilon\right)\right)}=\lim _{\substack{\left(\eta^{\prime}, \varepsilon\right) \rightarrow(\eta, 0) \\ \varepsilon>0}} \frac{N_{e}\left(\eta^{\prime}, \varepsilon\right)+\mathrm{const}}{N_{i}\left(\eta^{\prime}, \varepsilon\right)+\mathrm{const}}=\lim _{\substack{\left(\eta^{\prime}, \varepsilon\right) \rightarrow(\eta, 0) \\ \varepsilon>0}} \frac{N_{e}\left(\eta^{\prime}, \varepsilon\right)}{N_{i}\left(\eta^{\prime}, \varepsilon\right)}=\varphi\left(v_{\eta, 0}\right) .
$$

On the other hand, $h$ is a homeomorphism that sends $\mathcal{E}^{+}$to $\tilde{\mathcal{E}}^{+}$, hence

$$
\lim _{\substack{\left(\eta^{\prime}, \varepsilon\right) \rightarrow(\eta, 0) \\ \varepsilon>0}} \frac{\tilde{N}_{e}\left(h\left(\eta^{\prime}, \varepsilon\right)\right)}{\tilde{N}_{i}\left(h\left(\eta^{\prime}, \varepsilon\right)\right)}=\lim _{\substack{\left(\tilde{\eta}^{\prime}, \tilde{\varepsilon}\right) \rightarrow(\tilde{\tilde{\varepsilon}}, 0) \\ \tilde{\varepsilon}>0}} \frac{\tilde{N}_{e}\left(\tilde{\eta}^{\prime}, \tilde{\varepsilon}\right)}{\tilde{N}_{i}\left(\tilde{\eta}^{\prime}, \tilde{\varepsilon}\right)}=\varphi\left(\tilde{v}_{\tilde{\eta}, 0}\right) .
$$

This implies the second part of (12), so we proved Theorem 5, modulo Lemma 3.

\subsection{Simple diagrams}

Suppose that in the construction above, the polycycle $\gamma_{e}$ contains several polycycles $\gamma_{i}^{j}$, $j=1, \ldots, D$, monodromic from the interior. Suppose that assumptions of Theorem 5 hold for each pair of polycycles $\left(\gamma_{e}, \gamma_{i}^{j}\right)$. Then an unfolding of the corresponding vector field may have several invariant functions $\varphi_{j}$ simultaneously, one for each pair $\left(\gamma_{e}, \gamma_{i}^{j}\right)$.

Together with (13), this motivates the following definition.

Definition 17. A simple diagram of $\operatorname{rank}(d, D), d<D$, is an equivalence class of germs of smooth maps,

$$
\varphi:\left(M_{\varphi}, 0\right) \rightarrow\left(\mathbb{R}^{D}, \varphi(0)\right),
$$

where $\left(M_{\varphi}, 0\right)$ is a germ of a $d$-dimensional manifold. Two germs 22 are equivalent, $\varphi \sim \tilde{\varphi}$, provided that there exists a homeomorphism $\chi:\left(M_{\varphi}, 0\right) \rightarrow\left(M_{\tilde{\varphi}}, 0\right)$ such that

$$
\tilde{\varphi} \circ \chi=\varphi .
$$

Denote by $[\varphi]$ the equivalence class that contains $\varphi$.

Remark 8 . We write $\left(M_{\varphi}, 0\right)$ instead of $\left(\mathbb{R}^{d}, 0\right)$ to underline that the there are no canonical coordinates in the domain of $\varphi$, except for the origin. On the other hand, the coordinates in the image of the map 22 are well defined. These are the values of the functions $\varphi_{j}$.

Generic germ of map (22) has rank $d$. Two maps of rank $d$ are equivalent if and only if the germs of their images coincide. Fix a decomposition $\mathbb{R}^{D}=\mathbb{R}^{d} \oplus \mathbb{R}^{D-d}$. The first space is spanned by the first $d$ axis, the second one by the last $D-d$ ones.

Remark 9. For a generic smooth map $\varphi$, see $(22)$, its image is a graph of a map $f$ : $\left(\mathbb{R}^{d}, a\right) \rightarrow\left(\mathbb{R}^{D-d}, b\right),(a, b)=\varphi(0)$. This map is called the modulus of $\varphi$. Two generic smooth maps of the form (22) are equivalent iff their moduli coincide. So, we may call $f$ the modulus of the simple diagram $[\varphi]$. Any such modulus may be realized by some simple diagram.

For instance, a simple diagram $[\varphi]$ with generic $\varphi(22)$ for $d=1, D=2$ is characterized by a germ of a smooth function $(\mathbb{R}, a) \rightarrow(\mathbb{R}, b)$, cf. Theorem 2 .

Definition 14 in the form (13), and Definition 17 imply the following proposition. 
Proposition 4. Let $T$ be a Banach submanifold of $\operatorname{Vect}\left(S^{2}\right)$. Suppose that the moderate topological classification of families of class $T_{k}^{\dagger}$ possesses $D>d=k$ - $\operatorname{codim} T$ invariant functions $\varphi_{j}: T \rightarrow \mathbb{R}, j=1, \ldots, D$. Then the simple diagram $\left[\varphi_{V}\right]$, where $\varphi_{V}$ is given by $\varphi_{V}=\left(\varphi_{1}, \ldots, \varphi_{D}\right) \circ J_{V}$, or equivalently,

$$
\varphi_{V}(\alpha)=\left(\varphi_{1}\left(v_{\alpha}\right), \ldots, \varphi_{D}\left(v_{\alpha}\right)\right), \quad \varphi_{V}:\left(B\left(J_{V}\right), 0\right) \rightarrow\left(\mathbb{R}^{D}, \varphi_{V}(0)\right)
$$

is an invariant of this classification.

The following theorem is a local version of Theorem 3 .

Theorem 6. For any two positive integers $d<D$ there exists a Banach submanifold $\mathbf{T}_{D} \subset \operatorname{Vect}\left(S^{2}\right)$ of codimension $2 D+1$ such that the moderate topological classification of families of class $\left(\mathbf{T}_{D}\right)_{k}^{\dagger}$ has a simple diagram $[\varphi]$ of rank $(d, D)$ as an invariant. Any diagram with positive $\varphi(0)$ may be realized as an invariant of some family $V \in\left(\mathbf{T}_{D}\right)_{k}^{\dagger}$.

We prove this theorem in the next two subsections, then we deduce Theorem 3 from it. The proof is based on Theorem 5 and Proposition 4.

\subsection{Six parameter local families with a functional invariant}

In this section we prove Theorem 6 for $d=1, D=2$. Let us describe the class $\mathbf{T}_{2}$.

Consider a vector field $v_{0}$ with a hyperbolic polycycle $\gamma_{e}\left(v_{0}\right)$ with three vertexes: saddles $L_{1}, L_{2}, L_{3}$, and five edges: a separatrix loop of the saddle $L_{1}$, and four time oriented connections: $L_{j} L_{j+1}, L_{j+1} L_{j}, j=1,2$, see Figure 4 . This polycycle includes three smaller polycycles: the separatrix loop $\gamma_{i}^{1}$ of the saddle $L_{1}$, the polycycle $\gamma_{i}^{2}$ formed by the connections $L_{1} L_{2}, L_{2} L_{1}$, and the polycycle $\gamma_{h}$ formed by the connections $L_{2} L_{3}$, $L_{3} L_{2}$. Each of these three smaller polycycles is located outside of the other two. The polycycle $\gamma_{h}$ is a polycycle "heart", see Section 2.1.2.

The polycycle $\gamma_{e}$ is therefore monodromic from the exterior, and the polycycles $\gamma_{i}^{1}, \gamma_{i}^{2}$ are monodromic from the interior. Let $\lambda_{j}$ be the characteristic number of $L_{j}$. Then the characteristic numbers of the polycycles $\gamma_{i}^{1}, \gamma_{i}^{2}, \gamma_{e}$ are given by

$$
\lambda\left(\gamma_{i}^{1}\right)=\lambda_{1}, \quad \lambda\left(\gamma_{i}^{2}\right)=\lambda_{1} \lambda_{2}, \quad \lambda\left(\gamma_{e}\right)=\lambda_{1}^{2} \lambda_{2}^{2} \lambda_{3} .
$$

Suppose that both pairs $\left(\gamma_{e}, \gamma_{i}^{1}\right)$ and $\left(\gamma_{e}, \gamma_{i}^{2}\right)$ satisfy (14), that is:

$$
\lambda_{1}^{2} \lambda_{2}^{2} \lambda_{3}>1, \quad \lambda_{1}<1, \quad \lambda_{1} \lambda_{2}<1 .
$$

This completes the description of the polycycle $\gamma_{e}$. Note that both pairs $\left(\gamma_{e}, \gamma_{i}^{1}\right)$ and $\left(\gamma_{e}, \gamma_{i}^{2}\right)$ satisfy the properties formulated in Section 3.3.1. Hence, both classes $\hat{\mathbf{T}}\left(\gamma_{e}, \gamma_{i}^{1}\right)$ and $\hat{\mathbf{T}}\left(\gamma_{e}, \gamma_{i}^{2}\right)$ are well defined. Let $\mathbf{T}_{2}$ be the intersection of these two classes.

$$
\mathbf{T}_{2}=\hat{\mathbf{T}}\left(\gamma_{e}, \gamma_{i}^{1}\right) \cap \hat{\mathbf{T}}\left(\gamma_{e}, \gamma_{i}^{2}\right)
$$

This class has codimension 5 . 


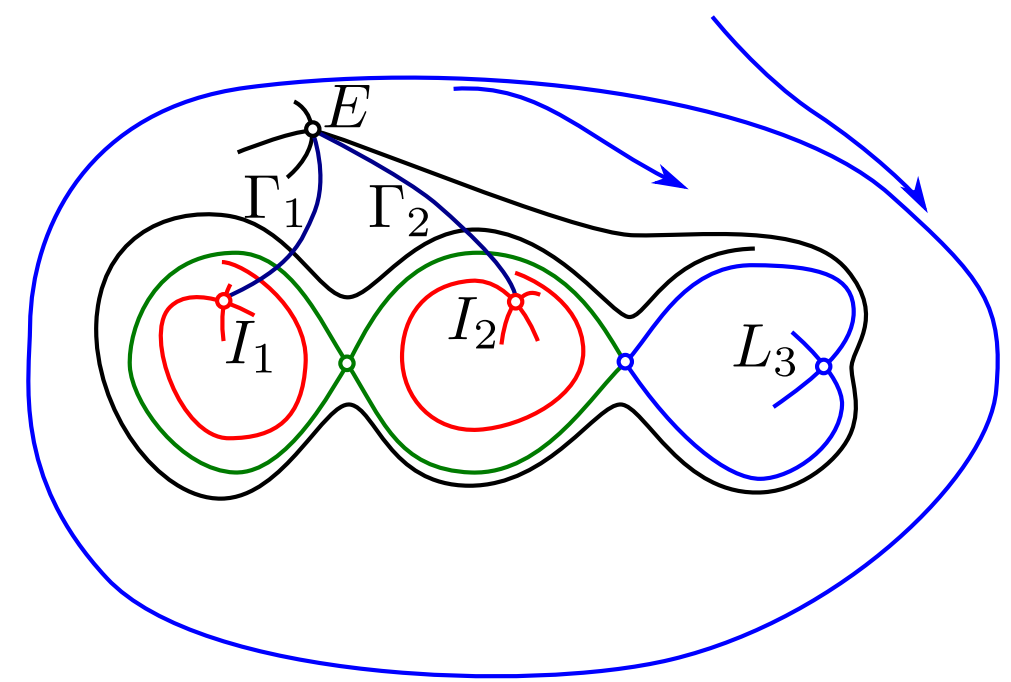

Figure 4: Phase portrait of a vector field of class $\mathbf{T}_{2,1}$. The phase portrait of a vector field of class $\mathbf{T}_{2}$ may lack the outer semi-stable limit cycle.

Remark 10. For $v \in \mathbf{T}_{2}$, the same saddle $E$ plays the role of $E$ from Section 3.3.2 for both pairs $\left(\gamma_{e}, \gamma_{i}^{1}\right)$ and $\left(\gamma_{e}, \gamma_{i}^{2}\right)$, though the saddles $I_{1}, I_{2}$ inside the polycycles $\gamma_{i}^{1}, \gamma_{i}^{2}$ are of course different, see Figure 4. In a neighborhood of $v \in \mathbf{T}_{2}$, we have $\mathbf{T}_{2}=\hat{\mathbf{T}}\left(\gamma_{e}, \gamma_{i}^{1}\right)=$ $\hat{\mathbf{T}}\left(\gamma_{e}, \gamma_{i}^{2}\right)$. Indeed, a vector field in $\hat{\mathbf{T}}\left(\gamma_{e}, \gamma_{i}^{1}\right)$ has the polycycle $\gamma_{e}$ preserved, hence both $\gamma_{i}^{1}$ and $\gamma_{i}^{2}$ are preserved, and a small perturbation cannot destroy (25).

Let $V$ be a local family of the class $\left(\mathbf{T}_{2}\right)_{6}^{\pitchfork}$, i.e., $v_{0} \in \mathbf{T}_{2}$, and $V$ is transverse to $\mathbf{T}_{2}$ at $v_{0}$. As $\mathbf{T}_{2}=\hat{\mathbf{T}}\left(\gamma_{e}, \gamma_{i}^{1}\right)$ near $v_{0}$, we can apply Theorem 5 to $\gamma_{i}=\gamma_{i}^{1}$, and get an invariant function $\varphi_{1}$ :

$$
\varphi_{1}: \mathbf{T}_{2} \rightarrow \mathbb{R}, \quad \varphi_{1}(w)=-\frac{\log \lambda\left(\gamma_{i}^{1}(w)\right)}{\log \lambda\left(\gamma_{e}(w)\right)}
$$

We can apply the same theorem to $\gamma_{i}=\gamma_{i}^{2}$, and get an invariant function

$$
\varphi_{2}: \mathbf{T}_{2} \rightarrow \mathbb{R}, \quad \varphi_{2}(w)=-\frac{\log \lambda\left(\gamma_{i}^{2}(w)\right)}{\log \gamma_{e}(w)}
$$

Therefore, due to Proposition 4, the simple diagram $\left[\varphi_{V}\right]$ given by $\varphi_{V}=\left(\varphi_{1}, \varphi_{2}\right) \circ J_{V}$ is an invaraint of the moderate topological classification of the families of class $\left(\mathbf{T}_{2}\right)_{6}^{\omega_{\mathrm{W}}}$.

The only restriction on the functions $\lambda_{j}\left(w_{\eta}\right)$ is $(25)$, hence any simple diagram $[\varphi]$ of rank $(1,2)$ with positive $\varphi(0)$ can be realized as $\left[\varphi_{V}\right]$. This proves Theorem 6 for $d=1$, $D=2$, modulo Lemma 3 .

\subsection{Simple diagrams as functional invariants}

The general version of Theorem 6 is proved in the same way. Fix $0<d<D$. Consider a vector field $v_{0}$ having a polycycle $\gamma_{e}$ with $D+1$ hyperbolic saddles $L_{1}, \ldots, L_{D+1}$, 
and $2 D+1$ time oriented edges: the loop of $L_{1}$ and the connections $L_{j} L_{j+1} ; L_{j+1} L_{j}$. The edges $L_{D} L_{D+1} ; L_{D+1} L_{D}$ form a polycycle of the type "heart", see Figure 3 . The characteristic numbers of the saddles $L_{j}$ are $\lambda_{j}$. The polycycle $\gamma_{i}^{1}$ is the loop of $L_{1}$, and the polycycles $\gamma_{i}^{j}, j=2, \ldots, D-1$ are formed by the edges $L_{j-1} L_{j} ; L_{j} L_{j-1}$. Their characteristic numbers are:

$$
\lambda\left(\gamma_{i}^{1}\right)=\lambda_{1}, \quad \lambda\left(\gamma_{i}^{j}\right)=\lambda_{j-1} \lambda_{j}, j>1 .
$$

The characteristic number of the "large" polycycle is

$$
\lambda\left(\gamma_{e}\right)=\lambda_{D+1} \prod_{1}^{D} \lambda_{j}^{2}
$$

We require:

$$
\lambda\left(\gamma_{i}^{j}\right)<1, \quad \lambda\left(\gamma_{e}\right)>1
$$

Let $\mathbf{T}_{D}$ be given by

$$
\mathbf{T}_{D}=\hat{\mathbf{T}}\left(\gamma_{e}, \gamma_{i}^{1}\right) \cap \ldots \cap \hat{\mathbf{T}}\left(\gamma_{e}, \gamma_{i}^{D}\right) .
$$

In a neighborhood of any vector field $v_{0} \in \mathbf{T}_{D}$, we have $\mathbf{T}_{D}=\hat{\mathbf{T}}\left(\gamma_{e}, \gamma_{i}^{j}\right)$, and $\mathbf{T}_{D}$ is a Banach submanifold of the space Vect $\left(S^{2}\right)$ of codimension $2 D+1$.

Let $V$ be a local family of class $\left(\mathbf{T}_{D}\right)_{k}^{\dagger}, k=d+\operatorname{codim} \mathbf{T}_{D}$. As $\mathbf{T}_{D}=\hat{\mathbf{T}}_{\gamma_{e}, \gamma_{i}^{j}}$ near $v_{0}$, Theorem 5 implies that functions

$$
\varphi_{j}: \mathbf{T}_{D} \rightarrow \mathbb{R}, \quad w \mapsto-\frac{\log \lambda\left(\gamma_{i}^{j}(w)\right)}{\log \lambda\left(\gamma_{e}(w)\right)}
$$

are invariant in sense of Definition 14,

Proposition 4 implies that the simple diagram $\left[\varphi_{V}\right]$ given by $\varphi_{V}=\left(\varphi_{1}, \ldots, \varphi_{D}\right) \circ J_{V}$ is a functional invaraint of moderate topological equivalence of families $V \in\left(\mathbf{T}_{D}\right)_{k}^{\dagger}$.

As the characteristic numbers $\lambda_{j}(w)$ may be chosen as arbitrary functions of parameters with the only restrictions $(26)$, the simple diagram $\left[\varphi_{V}\right]$ may be arbitrary with positive $\varphi(0)$. This proves Theorem 6 , modulo Lemma 3.

\subsection{Non-local families with functional invariants}

In this section we deduce Theorem 3 from Theorem 6 .

Let $V$ and $\tilde{V}$ be two local families of class $\left(\mathbf{T}_{D}\right)_{k}^{\dagger}$, and $\mathbf{V}, \tilde{\mathbf{V}}$ be their representatives. Note that moderate topological equivalence of non-local families $\mathbf{V}, \tilde{\mathbf{V}}$ does not imply the moderate topological equivalence of $V$ and $\tilde{V}$. Indeed, the corresponding homeomorphism of the bases may send 0 to another point of $\tilde{\mathbf{V}} \cap \mathbf{T}_{D}$, but Definition 9 requires it to send zero to zero.

In order to avoid this problem, we introduce a class $\mathbf{T}_{D, d} \subset \mathbf{T}_{D}$ of codimension $k$. Additionally to the parts of the phase portrait required by definition of $\mathbf{T}_{D}$, a vector 
field $v \in \mathbf{T}_{D, d}$ has $d$ semistable limit cycles of multiplicity 2 separating the polycycle $\gamma_{e}$ and the limit cycle at infinity. Clearly, $\operatorname{codim} \mathbf{T}_{D, d}=d+\operatorname{codim} \mathbf{T}_{D}=k$.

Let $\mathbf{T}_{D, d}^{\dagger}$ be the class of non-local $k$-parametric families of vector fields $V \subset \hat{\mathcal{H}}$ that intersect $\mathbf{T}_{D, d}$ at a single point, and contain no other vector fields orbitally topologically equivalent to vector fields of class $\mathbf{T}_{D, d}$. Consider two moderately topologically equivalent families $\mathbf{V}, \tilde{\mathbf{V}} \in \mathbf{T}_{D, d}^{\dagger}$. Let us shift coordinates in these families so that $v_{0}=\mathbf{V} \cap \mathbf{T}_{D, d}$, $\tilde{v}_{0}=\tilde{\mathbf{V}} \cap \mathbf{T}_{D, d}$. Let $h$ be the homeomorphism of the bases from (1). Due to the definition of $\mathbf{T}_{D, d}^{\dagger}, h$ sends zero to zero, hence the local families $\left(\mathbf{V}, v_{0}\right)$ and $\left(\tilde{\mathbf{V}}, \tilde{v}_{0}\right)$ are moderately topologically equivalent. Note that these families belong to $\left(\mathbf{T}_{D, d}\right)_{k}^{\dagger}$, hence they belong to $\left(\mathbf{T}_{D}\right)_{k}^{\pitchfork}$ as well.

Finally, let $\left[\varphi_{V}\right]$ be the simple diagram provided by Theorem 6 . For a non-local family $\mathbf{V}$, let $V$ be its germ at $\mathbf{V} \cap \mathbf{T}_{D, d}$. Then the simple diagram $\left[\hat{\varphi}_{\mathbf{V}}\right]=\left[\varphi_{V}\right]$ is an invariant of moderate topological equivalence of families $\mathbf{V} \in \mathbf{T}_{D, d}^{\dagger}$. Therefore, families of class $\mathbf{T}_{D, d}^{\dagger}$ have simple diagrams $(22)$ as invariant of moderate topological equivalence, and any diagram with $\varphi(0) \in \mathbb{R}_{+}^{D}$ may be realized as the invariant of a family $\mathbf{V} \in \mathbf{T}_{D, d}^{\dagger}$. Due to Remark 9, an open dense subset of $\mathbf{T}_{D, d}^{\dagger}$ has a smooth map $\left(\mathbb{R}_{+}^{d}, a\right) \rightarrow\left(\mathbb{R}_{+}^{d^{\prime}}, b\right)$ as a functional invariant of the moderate topological equivalence.

This completes the proof of Theorem 3 modulo Lemma 3.

\section{Asymptotics of sparkling saddle connections}

In this section we prove Lemmas 1 and 3 , and thus complete the proofs of Theorems $2-4$ and 6 .

\subsection{General asymptotics lemma and connection equation}

Lemma 1 follows from Lemma 3, see Remark 7, which, in turn contains two statements: one about the sparkling saddle connections related to the polycycle $\gamma_{i}$; another one about those related to the polycycle $\gamma_{e}$, see Section 2.1.2. These are two particular cases of one general lemma that will be stated now.

Consider a vector field $v$ having a hyperbolic monodromic polycycle $\gamma$. Let $\lambda(\gamma)$ be its characteristic number, see Definition 15.

Mark one edge of $\gamma$, say from $L$ to $M$, and a point $O$ on it. Suppose that $\lambda(\gamma)<1$, and there exists a hyperbolic saddle $I$ whose incoming separatrix $W_{I}^{s}$ winds toward $\gamma$ in the negative time. Let $\Gamma$ be a cross section through $O$ with one endpoint $I$ transversal to $v$ everywhere except for $I$. A germ of a monodromy map $\Delta_{\gamma}:(\Gamma, O) \rightarrow(\Gamma, O)$ is well defined by assumption that $\gamma$ is monodromic. Suppose that the germ $\Delta_{\gamma}$ may be extended to the monodromy map $\Delta_{\gamma}: I^{\prime} O \rightarrow I O$, where $I^{\prime}$ is the first intersection point of $\Gamma$ and the separatrix $W_{I}^{s}$ ran from $I$ in the negative time, see Figure 5a.

Let $\mathcal{E}$ be a $(d+1)$-parameter (non-generic) family of vector fields,

$$
\mathcal{E}=\left\{v_{\beta} \mid \beta \in\left(\mathbb{R}^{d+1}, 0\right)\right\}, \beta=(\eta, \varepsilon), \eta \in\left(\mathbb{R}^{d}, 0\right), \varepsilon \in(\mathbb{R}, 0), v_{0}=v .
$$




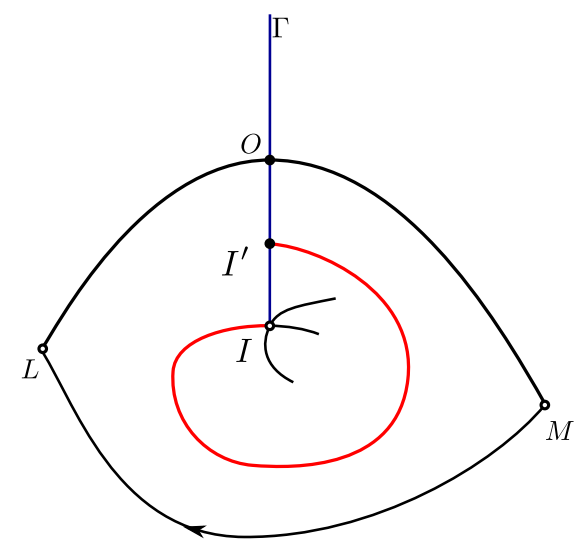

(a)

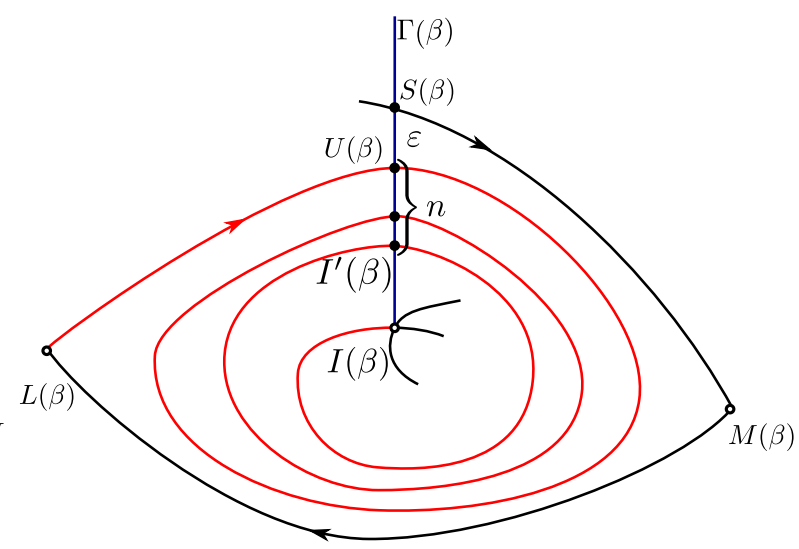

(b)

Figure 5: Geometry of the connection equation

Suppose that for $\varepsilon=0, \beta=(\eta, 0)$ the vector field $v_{\beta}$ has a polycycle $\gamma(\eta)$ continuously depending on $\eta ; \gamma(0)=\gamma$. Let $L(\beta), M(\beta), I(\beta)$ be hyperbolic saddles continuously depending on $\beta$ and coinciding with $L, M, I$ for $\beta=0$. For $\varepsilon \neq 0$ all the edges of the polycycle $\gamma$ stay unbroken except for the connection between $L(\beta)$ and $M(\beta)$.

For small $\eta$, the polycycle $\gamma(\eta)$ is hyperbolic; let $\lambda(\eta)=\lambda(\gamma(\eta))<1$ be its characteristic number. Let $U(\beta)(S(\beta))$ be the first intersection point of the unstable separatrix of $L(\beta)$ (stable separatrix of $M(\beta)$ ) with $\Gamma$, continuous in $\beta$; $U(0)=S(0)=O$. Consider a family of cross sections $\Gamma(\beta)$ that connect $S(\beta)$ and $I(\beta)$; assume that $\Gamma(\beta)$ coincides with $\Gamma$ outside a small neighborhood of $I$. Suppose that for small $\beta$, the map $\Delta_{\gamma}$ may be extended in $\beta$ as a map of an $\operatorname{arc}$ in $\Gamma$ to an $\operatorname{arc}$ in $\Gamma_{\beta}$ :

$$
\Delta_{\gamma, \beta}: I^{\prime}(\beta) S(\beta) \rightarrow I(\beta) U(\beta),
$$

see Figure 5b.

As before, say that $v_{\beta}$ has an $n$-winding connection $L(\beta) I(\beta)$ provided that the outcoming separatrix of $L(\beta)$ crosses the arc $\Gamma(\beta)$ with the point $I(\beta)$ excluded at exactly $n$ points, and enters $I(\beta)$.

Let $x$ be any smooth chart on $\Gamma$ positive on the semitransversal where the monodromy map $\Delta_{\gamma, 0}$ is defined. Let us make a reparametrization and a coordinate change similar to the previous ones:

$$
\varepsilon=x(U(\beta))-x(S(\beta)), \quad x_{\beta}=x-x(S(\beta)) .
$$

In this notation,

$$
x_{\beta}(S(\beta))=0, \quad x_{\beta}(U(\beta))=\varepsilon .
$$

Let $X(\beta):=x_{\beta}\left(I^{\prime}(\beta)\right)$. Then the connection equation

$$
\Delta_{\gamma, \beta}^{n-1}(\varepsilon)=X(\beta), \quad \beta=(\eta, \varepsilon),
$$

is equivalent to the fact that $v_{\beta}$ has an $n$-winding connection $L(\beta) I(\beta)$, see Figure $5 \mathrm{~b}$. 
Lemma 4. In assumption of this subsection, the connection equation has a unique solution $i_{n}(\eta)$ for any $n$ large enough defined on a neighborhood of 0 non-depending on $n$. The functional sequence $i_{n}$ is monotonically decreasing and

$$
\log \left(-\log i_{n}(\eta)\right)=-n \log \lambda(\eta)+O(1) .
$$

The upper bound for the remainder term in the right hand side is uniform in $\eta$.

This lemma is proved in the rest of this section.

\subsection{Local families of correspondence maps of hyperbolic saddles}

Here we recall a definition of the correspondence (Dulac) map of a hyperbolic saddle. Lemma 5 stated below claims that for a local family of such saddles the Dulac map behaves like $x^{\lambda(\beta)}$, where $\lambda(\beta)$ is the parameter depending characteristic value of the saddle. In case of a parameter depending linear hyperbolic saddle, $\dot{x}=x ; \dot{y}=-\lambda(\beta) y$, we have: $\Delta_{\beta}(x)=x^{\lambda(\beta)}$, and the lemma is trivial. Suppose now that $\lambda(0)$ is irrational, and $v_{\beta}$ is $C^{\infty}$ smooth. Then the corresponding local family is non-resonant. Smooth orbital normal form for this family is linear [8]. In this case the lemma is trivial again. The only non-trivial case is $\lambda(0) \in \mathbb{Q}$. The lemma for both cases simultaneously is proved at the end of this section.

Consider a local family of vector fields in the sphere with a hyperbolic saddle $L(\beta)$. Let $\lambda(\beta)$ be the characteristic number of $L(\beta)$. By a smooth parameter depending coordinate change we may put the saddle at the origin and two separatrixes of the saddle to positive coordinate rays. A parameter depending correspondence or Dulac map $\Delta_{\beta}$ is a map of a semitransversal $\Gamma^{+}$with a vertex on the incoming separatrix to a semitransversal $\Gamma^{-}$ with a vertex on the outgoing one along the orbits of the vector field of the family; the map is defined near the vertex of a semitransversal. Consider smooth charts $x, y$ on the semitransversals $\Gamma^{+}, \Gamma^{-}$that vanish at the vertexes. These charts will be called natural.

We shall formulate our estimates on $\Delta_{\beta}$ using $g=O(f)$ and $g=\Theta(f)$ notation. Let $f$ and $g$ be two functions $\mathbb{R}_{+} \rightarrow \mathbb{R}$. We write

$$
\begin{aligned}
& g=O(f) \quad \Leftrightarrow \quad \exists C>0 \exists \varepsilon>0 \forall x \in(0, \varepsilon):|g(x)| \leq C|f(x)| ; \\
& g=\Theta(f) \quad \Leftrightarrow \quad \exists 0<c<C \exists \varepsilon>0 \forall x \in(0, \varepsilon): c|f(x)| \leq|g(x)| \leq C|f(x)| .
\end{aligned}
$$

For parameter depending maps $f_{\beta}, g_{\beta}, \beta \in\left(\mathbb{R}^{k}, 0\right)$, the equalities $g_{\beta}=O\left(f_{\beta}\right)$ and $g_{\beta}=\Theta\left(f_{\beta}\right)$ mean the same relations as above with $c, C$ not depending on $\beta$.

In what follows, we use notation $D_{x}, D_{\varepsilon}$ for partial derivatives $\frac{\partial}{\partial x}, \frac{\partial}{\partial \varepsilon}$.

Lemma 5. Consider a local family $\left\{v_{\beta} \mid \beta \in\left(\mathbb{R}^{k}, 0\right)\right\}$ of vector fields with a hyperbolic saddle $L_{\beta}$, having the correspondence map $\Delta_{\beta}$. Let $\varepsilon$ be a component of $\beta, \varepsilon \in(\mathbb{R}, 0)$. Then in any natural charts $x, y$ on the semitransversals $\Gamma^{+}, \Gamma^{-}$, the map $y=\Delta_{\beta}(x)$ has the following properties:

$$
\begin{aligned}
\Delta_{\beta}(x) & =\Theta\left(x^{\lambda(\beta)}\right), \\
D_{x} \Delta_{\beta}(x) & =\Theta\left(x^{\lambda(\beta)-1}\right), \\
D_{\varepsilon} \Delta_{\beta}(x) & =O\left(x^{\lambda(\beta)} \log x\right) .
\end{aligned}
$$


in some neighborhood of 0 in $\mathbb{R}_{+} \times \mathbb{R}^{k}$. All the estimates are uniform in $\beta$ for $\beta$ small enough.

The collection of these three properties will be referred to as property (32).

Remark 11. If two families of maps, $\Delta_{\beta}$ and $\tilde{\Delta}_{\beta}$, satisfy property (32) with exponents $\lambda(\beta)$ and $\mu(\beta)$ respectively, then their composition satisfies this property with the exponent $\lambda(\beta) \mu(\beta)$. This follows from the chain rule, and may be checked by a straightforward calculation that we skip.

\subsection{Poincaré maps for unperturbed hyperbolic polycycles}

Here we prove that the Poincaré map of a parameter depending hyperbolic polycycle satisfies property (32).

Consider a vector field $v$ with a hyperbolic polycycle $\gamma$ monodromic from inside or from outside. Let $\Delta_{\gamma}$ be its monodromy map corresponding to a semitransversal $\Gamma^{+}$ with a vertex $O \in \gamma$, and $\lambda(\gamma)$ be the characteristic number of the polycycle. Let $\Gamma$ be a cross-section to $\gamma$ at $O$ such that $\Gamma \supset \Gamma^{+}$. Let $x:(\Gamma, O) \rightarrow(\mathbb{R}, 0)$ be a smooth chart on $\Gamma$ positive on $\Gamma^{+} \backslash O$.

Corollary 2. The monodromy map $\Delta_{\gamma}$ satisfies relations (32a), (32b) with $\lambda(\beta)$ replaced by $\lambda(\gamma)$.

Remark 12. This corollary, together with inequalities (2) (resp., (14)), implies that $O$ is an attracting fixed point for the Poincaré map $\Delta_{\gamma}$ (resp., $\Delta_{e}$ ), and a repelling fixed point for the Poincaré map $\Delta_{l}$ (resp., $\Delta_{i}$ ), where $\Delta_{\gamma}$ and $\Delta_{l}$ are the same as in (3) (resp., $\Delta_{e}$ and $\Delta_{i}$ are the same as in (15)).

Proof. The proof follows from Remark 11. More details are given in the proof of the next corollary that works for the present one as well.

Let us turn back to the local family $\mathcal{E}$ from Section 4.1 and consider vector fields with the polycycle $\gamma(\eta)$. Let $\lambda(\gamma(\eta))$ be its characteristic number. Put $O(\eta)=\gamma(\eta) \cap \Gamma$. Denote by $\Delta_{\gamma, \eta}$ the Poincaré map corresponding to $\gamma(\eta)$ written in the chart $x_{\eta}=$ $x-x(O(\eta))$.

Corollary 3. The monodromy map $\Delta_{\gamma, \eta}$ satisfies relations $32 \mathrm{a}$, $32 \mathrm{~b}$ with $x, \lambda(\beta)$ replaced with $x_{\eta}$ and $\lambda(\gamma(\eta))$.

Proof. The map $\Delta_{\gamma, \eta}$ is a composition of the correspondence maps for hyperbolic saddles, the vertexes of the polycycle. Each correspondence map satisfies $(32 \mathrm{a})$ and $(32 \mathrm{~b})$ due to Lemma 5. Thus the composition of these maps satisfies (32a) and (32b) with the exponent $\lambda(\beta)$ equal to the product of the exponents for individual maps, see Remark 11. 


\subsection{Poincaré maps for slightly perturbed hyperbolic polycycles}

Let us now consider vector fields of the family $\mathcal{E}$ for $\varepsilon \neq 0$. In the notation of Section 4.1, let $\Lambda(\beta)$ be the product of the characteristic values of all the saddles met on the way from $M(\beta)$ to $L(\beta)$ along the chain of unbroken saddle connections, including $M(\beta)$ and $L(\beta)$. By definition, $\Lambda(\eta, 0)=\lambda(\eta)$.

Corollary 4. In the settings above, in the chart $x=x_{\beta}$, see (28), we have

$$
\Delta_{\gamma, \beta}(x)=\varepsilon+\widetilde{\Delta}_{\gamma, \beta}(x),
$$

where $\widetilde{\Delta}_{\gamma, \beta}$ satisfies 32 , with $\lambda(\beta)$ replaced by $\Lambda(\beta)$.

Proof. The corollary follows from $(29)$ and the observation that the map $\widetilde{\Delta}_{\gamma, \beta}$ still is a product of correspondence maps for hyperbolic saddles. The rest of the proof proceeds as in Corollary 3.

\subsection{Simple bounds for the monodromy map $\Delta_{\gamma, \beta}$}

In the next subsection we complete the proof of the Asymptotic Lemma 4. For this we have to study the iterates of the map $\Delta_{\gamma, \beta}$. In this subsection we compare this map with a simpler one, namely, with a monomial $C x^{\lambda}$ for some $\lambda$ and $C$. The iterates of the latter map are easy to calculate. It is important that the comparison works in the domain

$$
U=\{(x, \beta) \mid x \geq \varepsilon>0\}
$$

for $x$ and $\beta=(\eta, \varepsilon)$ small. The lower bound for $x$ is motivated by the equality $\Delta_{\gamma, \beta}(0)=$ $\varepsilon$.

Lemma 6 (Comparison Lemma). Let $\Lambda(0)<1, U$ be the same as in (34). Then in the chart $x=x_{\beta}$ we have

$$
\begin{aligned}
& \Delta_{\gamma, \beta}(x)=\Theta\left(x^{\Lambda(\eta, 0)}\right) \\
& D_{x} \Delta_{\gamma, \beta}>2 ; \\
& D_{\varepsilon} \Delta_{\gamma, \beta}>0
\end{aligned}
$$

for $(x, \beta) \in U$ small. Recall that (35a) is, by definition, uniform in $\beta$.

Remark 13. Instead of 2 , we could take any constant greater than 1 in relation (35b): below we will prove that $D_{x} \Delta_{\gamma, \beta} \rightarrow \infty$ as $x \rightarrow 0$ uniformly in $\beta$.

Proof. Let us apply $D_{\varepsilon}$ to both sides of (33) from Corollary 4, then use (32c). We have:

$$
D_{\varepsilon} \Delta_{\gamma, \beta}(x)=1+D_{\varepsilon} \tilde{\Delta}_{\gamma, \beta}(x)=1+O\left(x^{\Lambda(\beta)} \log x\right)=1+o(1) .
$$

This implies (35c). Similarly,

$$
D_{x} \Delta_{\gamma, \beta}(x)=D_{x} \tilde{\Delta}_{\gamma, \beta}(x)=\Theta\left(x^{\Lambda(\beta)-1}\right) \rightarrow \infty \text { as } x \rightarrow 0 \text { uniformly in } \beta,
$$


since $\Lambda(0)<1$, and $\beta$ is small. Thus we proved (35b).

Finally, let us prove (35a). Due to (33) and (32a),

$$
\Delta_{\gamma, \beta}(x)=\varepsilon+\Theta\left(x^{\Lambda(\beta)}\right), \quad \beta=(\eta, \varepsilon) .
$$

For the first summand we have

$$
\varepsilon \leq x=o\left(x^{\Lambda(\eta, 0)}\right)
$$

since $0<\Lambda(\eta, 0)<c<1$ for $\eta$ small enough. For the second summand, we need to prove that

$$
x^{\Lambda(\eta, \varepsilon)}=\Theta\left(x^{\Lambda(\eta, 0)}\right) \text { as } x \rightarrow 0 \text { inside } U,
$$

or equivalently,

$$
x^{\Lambda(\eta, \varepsilon)-\Lambda(\eta, 0)}=\Theta(1) \text { as } x \rightarrow 0 \text { inside } U .
$$

Since $\Lambda$ is a smooth function, we have

$$
x^{\Lambda(\eta, \varepsilon)-\Lambda(\eta, 0)}=x^{O(\varepsilon)}=\left(x^{\varepsilon}\right)^{O(1)} .
$$

Note that for $(x, \beta) \in U, x<1$, we have $\varepsilon^{\varepsilon} \leq x^{\varepsilon} \leq 1$, and $\varepsilon^{\varepsilon} \rightarrow 1$ as $\varepsilon \rightarrow 0$. Therefore,

$$
x^{\Lambda(\eta, \varepsilon)-\Lambda(\eta, 0)}=(1+o(1))^{O(1)}=1+o(1) .
$$

This implies (35a).

\subsection{Sparkling saddle connections}

In this section we complete the proof of Lemma 4, that is, prove the existence, uniqueness and monotonicity in $n$ of the solutions of the connection equation (30).

\subsubsection{Existence}

The arguments below are based on the Comparison Lemma 6. Namely we replace the Poincaré map in the connection equation by a smaller (larger) map, whose iterates may be easily studied and for which the connection equation may be easily solved. These solutions provide the estimates for the actual solutions of the connection equation.

Let us pass to the detailed proof. Denote still by $U$ a small subdomain of $\{(x, \beta) \mid 0<\varepsilon \leq x\}$ where all the estimates of this lemma hold. More precisely, let

$$
K=\left\{\beta=(\eta, \varepsilon)|| \eta \mid<r, \varepsilon \in\left[0, \varepsilon_{0}\right]\right\}, \quad U=\{(x, \beta) \mid \beta \in K, \varepsilon \leq x \leq r\}
$$

for $\varepsilon_{0}, r$ small. By (35a), there exists $C>0$ such that

$$
e^{-C} x^{\Lambda(\eta, 0)}<\Delta_{\gamma, \beta}(x)<e^{C} x^{\Lambda(\eta, 0)},
$$

where $\beta=(\eta, \varepsilon),(x, \beta) \in U$. Fix a small $\eta$ and denote for simplicity

$$
\Lambda(\eta, 0)=\lambda, f_{-}(x)=e^{-C} x^{\lambda}, f_{+}(x)=e^{C} x^{\lambda} .
$$


The following estimates are obviously uniform in $\eta$ small; for this reason we omit the dependence of $\lambda$ on $\eta$ in notation. Each of the maps $f_{ \pm}$has two fixed points: 0 and $x_{ \pm}=\exp \left( \pm \frac{C}{1-\lambda}\right)$. Note that $x_{-}<1<x_{+}=x_{-}^{-1}$. On the segment $\left[0, x_{-}\right]$the map $f_{-}$ pushes all points from the repeller 0 to the attractor $x_{-}$. In particular, all the negative iterates of $f_{-}$are well-defined in $\left(0, x_{-}\right)$, hence for any $\tau \in\left(0, x_{-}\right)$, and any $n$ the equation $f_{-}^{n}(x)=\tau$ has a solution in $\left(0, x_{-}\right)$. Let $U$ be chosen so that in $U, x<x_{-}<x_{+}$.

Let $m \in \mathbb{N}, \tau \in\left(0, x_{-}\right)$and $\varepsilon$ be such that $f_{-}^{m}(\varepsilon)=\tau$, and $(\varepsilon, \beta) \in U$. Then

$$
\Delta_{\gamma, \beta}^{m}(\varepsilon)>\tau
$$

The right hand side of the connection equation (30) does not in general belong to $\left(0, x_{-}\right)$. Let us replace equation (30) by an equivalent one with the right hand side in $\left(0, x_{-}\right)$. For this let

$$
T(\beta)=x_{\beta}\left(\Delta_{\gamma, \beta}^{-a}\left(I^{\prime}(\beta)\right)\right) .
$$

Note that for $a$ sufficiently large, $T(\eta, 0)$ may be arbitrary small because the separatrix of $I(\eta, 0)$ approaches the connection $l$ in the negative time. Hence, for $r$ in the definition of $K$ sufficiently small, $a$ may be chosen so large that for any $\beta \in K, T(\beta)<x_{-}$. Connection equation (30) is equivalent to

$$
\Delta_{\gamma, \beta}^{n-a-1}(\varepsilon)=T(\beta), \beta \in K
$$

Let $m=n-a-1$. Take

$$
\psi_{m, \eta}(\varepsilon)=\Delta_{\gamma, \beta}^{m}(\varepsilon)-T(\beta), \beta=(\eta, \varepsilon) .
$$

Let $\varepsilon_{m}^{+}$be the solution of the equation

$$
f_{-}^{m}\left(\varepsilon_{m}^{+}\right)=\tau, \tau=\max _{K} T
$$

It exists because $\tau<x_{-}$. Then $\psi_{m, \eta}\left(\varepsilon_{m}^{+}\right)>0$, by $(36)$. Let $\varepsilon_{m}^{-}$be the solution of the equation

$$
f_{+}^{m}\left(\varepsilon_{m}^{-}\right)=t, t=\min _{K} T \text {. }
$$

It exists because $t<x_{-}<x_{+}$. Then $\varphi_{m, \eta}\left(\varepsilon_{m}^{-}\right)<0$. By the Intermediate Value Theorem, equation $\varphi_{m, \eta}(\varepsilon)=0$ which is equivalent to 30 has a solution $\varepsilon_{m}$, and

$$
\varepsilon_{m}^{-}<\varepsilon_{m}<\varepsilon_{m}^{+}
$$

\subsubsection{Uniqueness}

Let us prove that for $n$ large enough solution for (30) is unique. Suppose that $n$ is so large that we can replace (30) by (37). Then it is sufficient to prove that $D_{\varepsilon} \psi_{m, \eta}(\varepsilon)>0$ for large $m=n-a-1$ and sufficiently small $\varepsilon$. We have:

$$
D_{\varepsilon}\left(\Delta_{\gamma, \beta}^{m}(\varepsilon)\right)=\sum_{l=0}^{m-1}\left(D_{x} \Delta_{\gamma, \beta}^{l}\right)\left(\Delta_{\gamma, \beta}^{m-l}(\varepsilon)\right) \times D_{\varepsilon} \Delta_{\gamma, \beta}\left(\Delta_{\gamma, \beta}^{m-l-1}(\varepsilon)\right)+\left(D_{x} \Delta_{\gamma, \beta}^{m}\right)(\varepsilon) .
$$


Due to $(35 \mathrm{~b})$ and $(35 \mathrm{c})$, all the summands above are positive, and the last one is greater than $2^{m}$. Therefore, for $m$ large enough and $\varepsilon>0$ small enough, $D_{\varepsilon}\left(\Delta_{\gamma, \beta}^{m}(\varepsilon)\right)$ is greater than $\left|D_{\varepsilon} T\right|$, hence

$$
D_{\varepsilon} \psi_{m, \eta}(\varepsilon)>0
$$

\subsubsection{Monotonicity}

Let us prove that for $m$ large enough, $\varepsilon_{m}(\eta)<\varepsilon_{m-1}(\eta)$. By definition, for $\beta=\left(\eta, \varepsilon_{m}\right)$,

$$
\Delta_{\gamma, \beta}^{m}\left(\varepsilon_{m}\right)=T(\beta)
$$

Inequality $35 \mathrm{a}$ implies that for $\varepsilon>0, \Delta_{\gamma, \beta}(x)>x$. Hence,

$$
\Delta_{\gamma, \beta}^{m-1}\left(\varepsilon_{m}\right)<T(\beta) .
$$

Let $\psi_{m-1, \eta}$ be the same as in (38), with $m$ replaced by $m-1$. The latter inequality implies: $\psi_{m-1, \eta}\left(\varepsilon_{m}\right)<0$. By 42$), \psi_{m-1, \eta}(\varepsilon)$ monotonically increases in $\varepsilon$. By definition, $\psi_{m-1, \eta}\left(\varepsilon_{m-1}\right)=0$. Hence, $\varepsilon_{m-1}>\varepsilon_{m}$.

\subsubsection{Estimates}

Equations (39) and 40 may be easily solved; together with (41), the formulas for solutions imply (31). Recall that

$$
f_{-}(x)=e^{-C} x^{\lambda}, \quad x_{-}=e^{-\frac{C}{1-\lambda}}<1 .
$$

Note that

$$
f_{-}^{m}(x)=C_{m} x^{\lambda^{m}}, \quad C_{m}=e^{-C \frac{1-\lambda^{m}}{1-\lambda}} \searrow x_{-} \text {as } m \rightarrow \infty .
$$

For any $\tau<x_{-}$, equation $f_{-}^{m}(x)=\tau$ is equivalent to $x^{\lambda^{m}}=\tau C_{m}^{-1}$. Note that $\tau C_{m}^{-1} \nearrow$ $\tau x_{-}^{-1}<1$ as $m \rightarrow \infty$. Then, for the solution $\varepsilon_{m}^{+}$of $(39)$

$$
\log \left(-\log \varepsilon_{m}^{+}\right)=-m \log \lambda+\tau_{0}+o(1), \quad \tau_{0}=\log \left(-\log \tau x_{-}^{-1}\right) .
$$

Similarly, $f_{+}(x)=e^{C} x^{\lambda}, f_{+}^{m}(x)=C_{m}^{-1} x^{\lambda^{m}}$. Then, for the solution $x=\varepsilon_{m}^{-}$of 40,

$$
\log \left(-\log \varepsilon_{m}^{-}\right)=-m \log \lambda+t_{0}+o(1), \quad t_{0}=\log \left(-\log \tau x_{-}\right) .
$$

Note that $t_{0}>\tau_{0}$, because $x_{-}<1$. By 41

$$
-m \log \lambda+\tau_{0}+o(1)<\log \left(-\log \varepsilon_{m}\right)<-m \log \lambda+t_{0}+o(1) .
$$

This proves (31) and, together with it, Lemma 4. 


\subsection{Proof of asymptotic lemmas}

In this section we deduce Lemmas 1, 3 from Lemma 4. Lemma 1 follows from Lemma 3, so we prove the latter one only.

In statement (18), we deal with a polycycle $\gamma$ such that $\lambda(\gamma)<1$. Hence we can apply Lemma 4 and obtain (18). In order to prove the other half of Lemma 3, relation (19), it is enough to reverse the time, i.e., to replace the vector field $v$ by $-v$.

Let us now prove $(19)$ in more details. Consider a family $\mathcal{E}$ from Lemma 3. Let $\gamma(\eta)$ be the monodromic polycycle of the vector field $v_{(\eta, 0)}$. By assumption $(2), \lambda(\eta)>1$. By definition of the family $\mathcal{E}$, there exists a hyperbolic saddle $E$ whose separatrix winds to $\gamma_{e}(\eta)$ in the positive time. The family $\mathcal{E}$ from Lemma 3 is a particular case of the family (27) with the only difference: $\lambda(\gamma)>1$ instead of $\lambda(\gamma)<1$. Let us now reverse the time. We will get a vector field $-v_{(\eta, 0)}$ with the same polycycle, but with an opposite orientation. Denote it by $\gamma^{-}(\eta)$. The characteristic numbers of the saddles, under the time reversal, are replaced by the reciprocal ones. Hence, $\lambda\left(\gamma^{-}(\eta)\right)=\lambda^{-1}\left(\gamma_{e}(\eta)\right)<1$. The modified family $\mathcal{E}$ is a particular case of the family (27). Lemma 4 is now applicable; it implies:

$$
\log \left(-\log e_{n}(\eta)\right)=-n \log \left(\lambda^{-1}(\eta)\right)+O(1),
$$

This relation is equivalent to (19). This completes the proof of Lemma 3, hence Lemma 1 , modulo Lemma 5.

\subsection{Correspondence maps of hyperbolic saddles: proof of Lemma 5}

Preliminary considerations Here we prove Lemma 5. Let $\left\{v_{\beta}\right\}$ be a family of vector fields described in Lemma 5; $\beta$ is now the same as in Section 4.2. Clearly, the assertion of the lemma depends neither on the choice of $\Gamma^{+}$and $\Gamma^{-}$, nor on the choice of natural charts $x$ and $y$.

Let us choose some coordinates $\left(x_{\beta}, y_{\beta}\right)$ near $L(\beta)$ such that $S(\beta)=\left\{x_{\beta}=0\right\}$ and $U(\beta)=\left\{y_{\beta}=0\right\}$. Due to Hadamard-Perron Theorem, we may and will assume that $x_{\beta}$ and $y_{\beta}$ are $C^{3}$-smooth functions of the original coordinates and $\beta$. The differential of this coordinate change is $C^{2}$-smooth, thus $\dot{x}_{\beta}$ and $\dot{y}_{\beta}$ are $C^{2}$-smooth functions of $x_{\beta}$, $y_{\beta}$. For simplicity, we write $(x, y)$ instead of $\left(x_{\beta}, y_{\beta}\right)$. Since $\dot{x}(0, y)=0$ and $\dot{y}(x, 0)=0$, due to Hadamard Lemma, we have

$$
\dot{x}=x f_{1}(x, y, \beta) ; \quad \dot{y}=-y f_{2}(x, y, \beta),
$$

where $f_{1}$ and $f_{2}$ are $C^{1}$-smooth functions. This vector field has the same correspondence map as the vector field

$$
\begin{aligned}
& \dot{x}=x ; \\
& \dot{y}=-y g(x, y, \beta),
\end{aligned}
$$

where $g(x, y, \beta)=\frac{f_{2}(x, y, \beta)}{f_{1}(x, y, \beta)}$ is a $C^{1}$-smooth function as well. Clearly, $g(0,0, \beta)=\lambda(\beta)$. Choose a neighborhood $U \subset \mathbb{R}^{2} \times \mathbb{R}^{k}$ of $(L(0), 0)$ such that in $U$

$$
\frac{1}{2} \lambda(0)<g(x, y, \beta)<2 \lambda(0) \text {. }
$$


Choose $\Gamma^{+}$and $\Gamma^{-}$so that all trajectories of $v_{\beta}$ going from $\Gamma^{+}$to $\Gamma^{-}$stay in $U$. After a rescaling, we may and will assume that $\Gamma^{+}=\{y=1\}$ and $\Gamma^{-}=\{x=1\}$. We shall prove (32) for the restrictions of $x$ and $y$ to $\Gamma^{+}$and $\Gamma^{-}$, respectively.

Estimate of $\Delta_{\beta}$ This estimate follows the proof of Lemma 1 in [3], see also [5, Section 9.3].

Fix a small positive $x_{0}$. Consider a trajectory $(x, y)(t)$ of 43 starting at $\left(x_{0}, 1\right)$. In our notation, we skip the indication of the dependence of the solution on the parameter $\beta$. Due to 43a), we have $x(t)=x_{0} e^{t}$, hence this trajectory arrives to $\Gamma^{-}$at $T=-\log x_{0}$. Next, (44) implies that $y(t) \leq e^{-\lambda(0) t / 2}$, but we need a sharper estimate. Note that for $0 \leq t \leq T$ we have

$$
\int_{0}^{t} O(x(\tau))+O(y(\tau)) d \tau=\int_{0}^{t} O\left(e^{\tau-T}\right)+O\left(e^{-\lambda(0) \tau / 2}\right) d \tau=O(1) .
$$

Therefore, for $0 \leq t \leq T$ we have

$$
\begin{aligned}
\log y(t) & =\int_{0}^{t} \frac{\dot{y}(\tau)}{y(\tau)} d \tau=-\int_{0}^{t} g(x(\tau), y(\tau), \beta) d \tau \\
& =-\int_{0}^{t}(\lambda(\beta)+O(x(\tau))+O(y(\tau))) d t=-\lambda(\beta) t+O(1) .
\end{aligned}
$$

Thus

$$
y(t)=\Theta\left(e^{-\lambda(\beta) t}\right) .
$$

From now on replace $x_{0}$ by $x$. For $t=T=-\log x$, equation (46) implies (32a), because $y(T)=\Delta_{\beta}(x), e^{-\lambda(\beta) T}=x^{\lambda(\beta)}$.

Decomposition of $\Delta_{\beta}$ In order to estimate the derivatives of the germ of $\Delta_{\beta}$ at $x$, let us decompose it in the following way. Consider an auxiliary local cross-section $\Gamma^{x} \subset$ $\{(x, y), y \in(\mathbb{R}, 1)\} ;$ it passes through the point $(x, 1)$ and is orthogonal to $\Gamma^{+}$. Equip $\Gamma^{x}$ with the chart $y$. Let

$$
\begin{aligned}
& \Delta_{\beta}^{+}:\left(\Gamma^{+}, x\right) \rightarrow\left(\Gamma^{x}, 1\right), \\
& \Delta_{\beta}^{x}:\left(\Gamma^{x}, 1\right) \rightarrow\left(\Gamma^{-}, \Delta_{\beta}(x)\right) ;
\end{aligned}
$$

be the correspondence maps along the orbits of $v_{\beta}$, same as correspondence maps for $43 \mathrm{a}$, 43b). Then

$$
\Delta_{\beta}=\Delta_{\beta}^{x} \circ \Delta_{\beta}^{+}
$$

Estimate of $D_{x} \Delta_{\beta}(x)$ Note that $D_{x} \Delta_{\beta}^{+}(x)=\frac{g(x, 1, \beta)}{x}=\Theta\left(x^{-1}\right)$, hence it is enough to show that $\left.D_{y} \Delta_{\beta}^{x}(y)\right|_{y=1}=\Theta\left(x^{\lambda(\beta)}\right)$.

Recall that $x(t)=x(0) e^{t}$. Equation (43b) with $x$ replaced by $x(t)=x(0) e^{t}$, is a one-dimensional non autonomous equation. Let $y(t, \beta, a)$ be a solution of this equation 


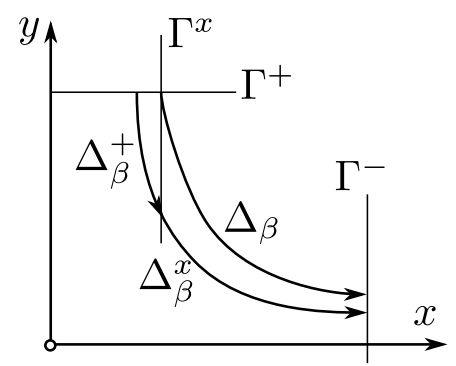

Figure 6: A saddle, some cross-sections and correspondence maps

with the initial condition $y(0, \beta, a)=a$; as before, $\beta=(\eta, \varepsilon)$. Denote by $z(t)$ and $w(t)$ the derivatives of this solution with respect to the initial conditions and the parameter $\varepsilon$ :

$$
z(t)=\left.D_{a} y(t, \beta, a)\right|_{a=1}, w(t)=D_{\varepsilon} y(t, \beta, 1), \varepsilon \in(\mathbb{R}, 0) .
$$

This implies: $z(0)=1, w(0)=0$. Denote for simplicity $y(t, \beta, 1)=y(t)$; we skip in this notation the dependence on $\beta$ that actually takes place. Note that

$$
\Delta_{\beta}(x)=y(T), T=-\log x ; D_{y} \Delta_{\beta}^{x}(1)=z(T), D_{\varepsilon} \Delta_{\beta}^{x}(1)=w(T) .
$$

The variational equation for $z(t)$ has the form:

$$
\dot{z}=-A(t) z, A(t)=\left(D_{y}(y g)\right)(x(t), y(t), \beta) .
$$

Hence, $A(t)=g(x(t), y(t), \beta)+y D_{y} g(x(t), y(t), \beta)=\lambda(\beta)+O(x(t))+O(y(t))$. Therefore, by 45 ,

$$
\log z(t)=-\int_{0}^{t} A(\tau) d \tau=-\lambda(\beta) t+O(1) ; \log z(T)=\lambda(\beta) \log x+O(1) .
$$

Hence, $\left(D_{y} \Delta_{\beta}^{x}\right)(1)=z(T)=\Theta\left(x^{\lambda(\beta)}\right)$, thus $D_{x} \Delta_{\beta}(x)=\Theta\left(x^{\lambda(\beta)-1}\right)$. This implies 32b .

Estimate of $D_{\varepsilon} \Delta_{\beta}(x)=w(T)$ The equation of variations for $43 \mathrm{~b}$ with respect to the parameter takes the form

$$
\dot{w}=-A(t) w-B(t), \quad B(t)=\left(D_{\varepsilon}(y g)\right)(x(t), y(t), \beta), \quad w(0)=0,
$$

$A(t)$ is the same as above. We have:

$$
w(t)=z(t) C(t), \quad \dot{C}=-B(t) z^{-1}(t), \quad w(0)=0 .
$$

Hence,

$$
w(T)=-z(T) \int_{0}^{T} B(t) z^{-1}(t) d t .
$$

We have: $B(t)=\left(D_{\varepsilon}(y g)\right)(x(t), y(t), \beta)=-y O(1), z^{-1}(t)=O(1) \exp (\lambda(\beta) t)$. Due to (46), $\exp (\lambda(\beta) t)=\Theta\left(y(t)^{-1}\right)$, hence $z^{-1}(t)=O\left(y(t)^{-1}\right)$. Thus

$$
D_{\varepsilon} \Delta_{\beta}(x)=w(T)=\Theta\left(x^{\lambda(\beta)}\right) \int_{0}^{T} O(1) d t=O\left(x^{\lambda(\beta)} \log x\right) .
$$


This implies $32 \mathrm{c}$.

Lemma 5 is proved. It implies Lemma 4. The proof of the main results of this paper is now completed.

\section{New perspective}

\subsection{No structural stability in codimension three}

Theorem 4 implies that there is an open set in the space of three parameter families of vector fields that are not structurally stable. Namely, consider two vector fields $v_{0}, \tilde{v}_{0}$ and their unfoldings that satisfy the assumptions of Section 2. If the ratios of the logarithms of the characteristic numbers of the saddles $L$ and $M$ for these vector fields are different, then the two unfoldings are not moderately topologically equivalent, however close they are.

\subsection{No versal families whose dimension equals the codimension of the degeneracy: a conjecture}

In early 70's Arnold suggested a new approach that revolutionized the bifurcation theory. It was based on a concept of versal families. The original definition of these families may be found in [2], and the final one in [1]. Without reproducing this definition we will mention only that versal families are special unfoldings of a degeneracy of a certain class that contain a very concentrated information about the bifurcations in arbitrary local families that unfold the degeneracies of this class. Since early 70's many versal families were investigated. It so happened that their dimension was always equal to the codimension of the degeneracy, though this is not required by the definition. Moreover, two different versal deformations of the same vector field are weakly (and even moderately) topologically equivalent. This equivalence holds for all versal deformations studied up to now. This is a folklore fact, not written anywhere.

Theorem 2 implies that two generic 6-parameter local families passing through the same vector field of class $\mathbf{T}_{2,1}$, are not topologically equivalent. Indeed, consider two generic unfoldings $V$ and $W$ of the same vector field of class $\mathbf{T}_{2,1}$. These families belong to an open subset of $\mathcal{V}_{6}\left(S^{2}\right)$ mentioned in Theorem 2. By this theorem, they may have different functional invariants $f:\left(\mathbb{R}_{+}, a\right) \rightarrow\left(\mathbb{R}_{+}, b\right), g:(\mathbb{R}, a) \rightarrow\left(\mathbb{R}_{+}, b\right)$, because any such germ may be realized as an invariant of such a local family. Hence, the families $V$ and $W$ are not equivalent.

This gives a strong evidence to the following

Conjecture 1. There are no versal 6-parameter local families that unfold generic vector fields of class $\mathbf{T}_{2,1}$. Moreover, for any $k \geq 6$ there exists an open set of vector fields for which there are no $k$-parameter versal families.

The second author has a strategy of the proof of this conjecture. Note that our arguments imply no functional invariants for seven-parametric unfoldings of vector fields 
of class $\mathbf{T}_{2,1}$. The problem of existence of versal families whose dimension is higher than the codimension of the degeneracy is still open.

Problem 1. Let $\mathbf{M} \subset \operatorname{Vect}\left(S^{2}\right)$ be a Banach submanifold of finite codimension. Is it true that for a generic $v \in \mathbf{M}$ and $k \geq$ codim $\mathbf{M}$ large enough, there exists a $k$-parameter versal deformation of $v$ ?

The authors expect that there exist classes $\mathbf{M}$ for which the answer is negative.

\subsection{Good, bad and ugly families of vector fields}

The space of all finite-parameter families of vector fields on the sphere may be split in three classes, each one more complicated than the previous class.

Good families are moderately structurally stable ones.

Conjecture 2. Generic one-parameter families of vector fields in the two sphere are good.

More difficult is

Conjecture 3. Generic two-parameter families of vector fields in the two sphere are good.

We do not expect that the proof of this conjecture is either easy or short. Plausibly, it requires a topological classification of all different classes of two parameter families. This classification is discussed below.

Bad families are those whose moderate topological classification has numeric invariants. These families are not structurally stable.

Ugly families are those whose moderate topological classification has functional invariants.

Study of the boundaries between good, bad and ugly families is a challenging problem. A particular statement is:

Problem 2. Distinguish structurally unstable generic three parameter families from the structurally stable ones.

In this paper we proved that bad three-parameter families exist. No doubt that good three-parameter families exist too.

An interesting problem is to construct more examples of bad three-parameter families.

Problem 3. What is the smallest number of parameters for which ugly families exist?

Theorem 3 implies that this number is no greater than 6 . We expect that the actual answer is even smaller. 


\subsection{Classification problems}

Problem 4. Classify all generic one-parameter local families of vector fields on the two-sphere up to moderate topological equivalence.

Preliminary steps of this classification, in particular, the classification of all the possible degeneracies, are done in [11]. A complete description, without a proof, is suggested in [7]. Together with his students N. Solodovnikov and V. Starichkova, the first author completes the justification of this description. As a by product, this description implies Conjecture 2.

Problem 5. Classify all generic two-parameter local families of vector fields on the two-sphere up to moderate topological equivalence.

This problem is far from being solved. The strategy may be the following. In [9], a complete list of polycycles that may occur in generic two and three parameter families was presented. It looks natural to study "sparkling saddle suspensions" over these polycycles. This construction may produce about two or three dozens of infinite series of two-parameter local families, each one corresponding to suspensions of sparkling saddle connections over the polycycles of codimension two from the list, or over two coexisting polycycles of codimension one. Two families in a series differ by a number and a mutual location of the separatrixes of saddles that form sparkling saddle connections. There may be other invariants. The families in each series should be classified like it is done for one-parameter families.

Complete classification of generic three-parameter families looks like a very large, but not yet hopeless problem. One of the first steps may be the following.

Problem 6. Find ALL the topological invariants of the family described in Theorem 4.

\subsection{Continuum of germs of bifurcation diagrams}

Arnold conjectured [1] that for any $k$ there exists but a finite number of pairwise topologically nonequivalent germs of bifurcation diagrams that may occur in generic $k$-parameter families. (A bifurcation diagram is a subset of the base of the family that corresponds to structurally unstable vector fields.) This conjecture was disproved in $[9]$ where a countable number of nonequivalent germs of bifurcation diagrams in three-parameter families was constructed. Recently it occurred that this effect may be observed even in two-parameter families [7].

Conjecture 4. There exists an open set in the space of three-parameter families such that the set of pairwise topologically different germs of bifurcation diagrams that may occur in the families from this set has cardinality continuum.

The first two authors have a strategy of the proof of this conjecture. 


\subsection{Numeric and functional invariants in local and semilocal bifurcations}

Semilocal theory studies bifurcations in a neighborhood of an arbitrary polycycle; denote this polycycle by $\gamma$. The phase space is now a germ of a neighborhood $\left(\mathbb{R}^{2}, \gamma\right)$. Moderate topological equivalence of semilocal families on $\left(\mathbb{R}^{2}, \gamma\right) \times\left(\mathbb{R}^{k}, 0\right)$ is defined as in Section 1 .

Problem 7. Are there numeric or functional invariants of moderate topological classification of semilocal families?

Problem 8. The same question about the local families: may numeric or functional invariants occur in the moderate topological classification of families of vector fields in a neighborhood of a singular point?

We expect that "hidden" sparkling saddle connections may occur in the unfoldings of polycycles, even hyperbolic, and produce numeric and functional invariants. The same expectation concerns unfoldings of complex singular points. This is a realization of the following heuristic principle:

All effects observed for global families in the plane may be observed for the local and semi-local ones, may be, with a greater number of parameters.

Acknowledgments The authors are grateful to Christian Bonatti, Anton Gorodetski and Alexei Klimenko for fruitful suggestions.

\section{References}

[1] V. I. Arnold, V. S. Afrajmovich, Y. S. Ilyashenko, and L. P. Shilnikov. Dynamical Systems V. Bifurcation Theory and Catastrophe Theory. Encyclopaedia of Mathematical Sciences. Berlin: Springer-Verlag, viii+271 pp. DOI: 10.1007/978-3-64257884-7. URL: http://www.springer.com/gp/book/9783540181736. Trans. of V. I. Arnold, V. S. Afrajmovich, Yu. S. Ilyashenko, and L. P. Shilnikov. Teorija bifurcacij. Russian. Dinamicheskije sistemy V. 1986.

[2] V. I. Arnold. "Lectures on bifurcations in versal families". In: Russian Mathematical Surveys 27.5 (1972), pp. 54-123. DOI: 10.1070/RM1972v027n05ABEH001385. URL: http://stacks .iop.org/0036-0279/27/i=5/a=A02.

[3] T. I. Golenishcheva-Kutuzova and V. A. Kleptsyn. "Convergence of the Krylov-Bogolyubov Procedure in Bowan's Example". In: Math. Notes 82 (5 2007), pp. 608-618. DOI: 10.1134/S0001434607110041.

[4] N. B. Goncharuk and Yu. S. Ilyashenko. "Large bifurcation supports". In preparation.

[5] J. H. Hubbard and B. H. West. Differential Equations: A Dynamical Systems Approach. Higher-Dimensional Systems. Texts in Applied Mathematics 18. NewYork: Springer-Verlag, 1995. ISBN: 0-387-94377-3. 
[6] Yu. Ilyashenko. "Centennial history of Hilbert's 16th problem". In: Bulletin (new series) of the American Mathematical Society 39.3 (2002), pp. 301-354. Dor: 10. 1090/S0273-0979-02-00946-1.

[7] Yu. S. Ilyashenko. "Towards the general theory of global planar bifurcations". In: Mathematical Sciences with Multidisciplinary Applications. In Honor of Professor Christiane Rousseau. And In Recognition of the Mathematics for Planet Earth Initiative. Ed. by B. Toni. Vol. 157. Springer Proceedings in Mathematics \& Statistics. Springer, 2016, pp. 269-299. ISBN: 978-3-319-31321-4. DOI: 10.1007/978-3319-31323-8.

[8] Yu. S. Ilyashenko and S. Yakovenko. "Finitely-smooth normal forms of local families of diffeomorphisms and vector fields". In: Russian Mathematical Surveys 46.1 (1991), pp. 3-39. URL: http://stacks.iop.org/0036-0279/46/i=1/a=R01.

[9] A. Kotova and V. Stanzo. "On few-parameter generic families of vector fields on the two-dimensional sphere". In: Concerning the Hilbert 16th problem. Vol. 165. American Mathematical Society Translation Series 2. Providence, RI: Amer. Math. Soc., 1995, pp. 155-201.

[10] R. Roussarie. "Weak and continuous equivalences for families on line diffeomorphisms". English. In: Dynamical systems and bifurcation theory, Proc. Meet. (Rio de Janeiro/Braz. 1985). Pitman Res. Notes Math. Ser. 160. 1987, pp. 377-385.

[11] J. Sotomayor. "Generic one-parameter families of vector fields on two-dimensional manifolds". In: Publications mathematiques de l'IHES 43 (1974), pp. 5-46. DOI: 10.1007/BF02684365 